Proceedings of International Mathematical Sciences

ISSN:2717-6355, URL:HTTPS://DERGIPARK.ORG.TR/TR/PUB/PIMS

Volume II Issue 2 (2020), Pages 103-128. Doi: 10.47086/Pims.827108

\title{
DIOPHANTINE ATTACK ON PRIME POWER WITH MODULUS
}

$$
N=p^{r} q
$$

\author{
SAIDU ISAH ABUBAKAR*, ZAID IBRAHIM**, SADIQ SHEHU *** AND AHMAD \\ RUFAI**** \\ *DEPARTMENT OF MATHEMATICS, SOKOTO STATE UNIVERSITY , SOKOTO, \\ NIGERIA. ORCID NUMBER:0000-0002-0201-0064: \\ **DEPARTMENT OF MATHEMATICS, SOKOTO STATE UNIVERSITY, SOKOTO, \\ NIGERIA. ORCID NUMBER:0000-0002-0251-6495: \\ ***DEPARTMENT OF MATHEMATICS, SOKOTO STATE UNIVERSITY, SOKOTO, \\ NIGERIA. ORCID NUMBER: 0000-0001-5908-7452: \\ ****DEPARTMENT OF MATHEMATICS, SOKOTO STATE UNIVERSITY, SOKOTO, \\ NIGERIA. ORCID NUMBER:0000-0003-3223-9924:
}

\begin{abstract}
The importance of keeping information secret cannot be overemphasized especially in today,s digital world where eavesdroppers are rampant in our chanels of communication. This made the use of strong encryption schemes inevitable in order to safeguard the security of our system. RSA cryptosystem and its variants have been designed to provide confidentiality and integrity of data in our medium of communication. This paper reports new short decryption exponent attack on prime power with modulus $N=p^{r} q$ for $r \geq 2$ using continued fraction method which makes it vulnerable to Diophantine attack and breaks the security of the cryptosystem by factoring the modulus into its prime factors since the hardness relies on the integer factorization problem. The paper also shows that if the short decryption exponent $d<\frac{1}{\sqrt{2}} \sqrt{N-2^{\frac{2 r+1}{r+1}} N^{\frac{r}{r+1}}}$, then one of the convergents $\frac{k}{d}$ can be found from the continued fraction expansion of $\frac{e}{N-\left[2^{\frac{2 r+1}{r+1}} N^{\frac{r}{r+1}}\right]}$ which leads to the successful factorization of prime power modulus $N=p^{r} q$ in polynomial time. The second part of the paper presents new findings on simultaneous factorization of $t$ prime power with moduli $N_{s}=p_{s}^{r} q_{s}$ for $s=1, \ldots, t$ using simultaneous Diophantine approximations and lattice basis reduction methods which produces the prime factors of the form $\left(p_{s}, q_{s}\right)$ for $s=1, \ldots, t$ in polynomial time where solutions of four system of equations of the form $e_{s} d-k_{s} \phi\left(N_{s}\right)=1$, $e_{s} d_{s}-k \phi\left(N_{s}\right)=1, e_{s} d-k_{s} \phi\left(N_{s}\right)=z_{s}$ and $e_{s} d_{s}-k \phi\left(N_{s}\right)=z_{s}$ are provided. Our results increases the short decryption exponent bounds of some reported works.
\end{abstract}

2020 Mathematics Subject Classification. 11A52 ; 11A54; 11A55.

Key words and phrases. Diophantine; Attacks; Prime Power; Modulus; Continued Fraction. (C)2020 Proceedings of International Mathematical Sciences.

Submitted November 17th, 2020. Published on december 30th, 2020. Communicated by Sahin UYAVER. 


\section{INTRODUCTION}

The RSA cryptosystem invented by Rivest, Shamir and Adleman is considered to be the most widely used public key cryptosystem in today's digital world, [1]. Since then it has being extensively used for many applications in government as well as commercial domains which include e-banking, secure telephone, smart cards and communications in different types of Networks 2 .

The security of this cryptosystem relies on the integer factorization problem. This cryptosystem has also many variants for computational efficiency. In this paper, we will focus on one of the variants known as prime power RSA with modulus $N=p^{r} q$ for $r \geq 2$. Fujioka et al. was the first to use the modulus $N=p^{2} q$ for digital signature whose computational speed is faster than the original RSA scheme, as reported in [3]. Also in 1998, Okamoto et al. proposed a public key cryptography scheme whose security is considered to be as difficult as factoring an RSA modulus of the form $N=p^{2} q$, as reported [4].

This paper focuses on the first variant given as $e d \equiv 1\left(\bmod p^{r-1}(p-1)(q-1)\right)$ Using the first prime power RSA variant, Takagi in 1999 proposed a fast CRT-RSA variant with modulus $N=p^{r} q$ which is considered to be less vulnerable to attacks than the original RSA scheme, [5]. Takagi (1999) showed that when $d<N^{\frac{1}{2(r+1)}}$ for $r \geq 2$, the modulus $N=p^{r} q$ can be factored efficiently using lattice based technique. May (2004), reported an improvement on the bound of Takagi, where he showed that the modulus $N=p^{r} q$ is insecure if the short secret exponent $d<N^{\max \left\{\frac{r}{(r+1)^{2}}, \frac{(r-1)^{2}}{(r+1)^{2}}\right\}}$ using generalized Coppersmith's method, as reported by 6]. Also, Sarkar (2014) reported the used of small secret exponent attack on prime power RSA with modulus $N=p^{2} q$ where he proved that the cryptosystem is insecure if the decryption exponent bound $d<N^{0.395}$, 77. Furthermore, Lu et. al (2015) improved May's bound to $d<N^{\frac{r(r-1)}{(r+1)^{2}}}$ by method of lattice construction, [8. In another result, Sarkar (2016) reported an improved bound of Lu et al. for $2 \leq r \leq 4,9$.

For the second variant of prime power modulus $N=p^{r} q$, Itoh et al. (2008) showed that the prime factors of the prime power RSA modulus $N=p^{r} q$ can be found in polyomial time if the bound $d<\frac{2-\sqrt{2}}{r+1},[10$.

Also, Blomer and May (2004) reported generalized Wiener's attack using combination of continued fraction and lattice basis reduction techniques which showed that RSA modulus $N=p q$ is insecure when there exist some unknown integers $x, y, z$ such that equation $e x-y \phi(N)=z$ is satisfied where $x<\frac{1}{3} N^{\frac{1}{4}}$ and $|z|<e x N^{\frac{-3}{4}}$, [11. In his work, Hinek (2007) proved that $k$ instances of RSA moduli $N_{i}$ can be factored if $d<N^{\gamma}$ for $\gamma=\frac{k}{2(k+1)}-\varepsilon$ where $\varepsilon$ is a small constant determine based on the size of $\max \left\{N_{i}\right\}$, as reported in [12].

In another development, Nitaj et al. (2014) presented two scenarios which showed that $k$ instances of RSA moduli $N_{i}=p_{i} q_{i}$ can be factored simultaneously in polynomial time using simultaneous Diophantine approximation and LLL algorithm , 13. In the first scenario, they showed that if the equation $e_{i} x-y_{i} \phi\left(N_{i}\right)$ is satisfied where $x<N^{\delta}, y_{i}<N^{\delta},\left|z_{i}\right|<\frac{p_{i}-q_{i}}{3\left(p_{i}+q_{i}\right)} y_{i} N^{\frac{1}{4}}$ for $\delta=\frac{k}{2(k+1)}, N=\min \left\{N_{i}\right\}$ then $k$ RSA moduli can be factored simultaneously. For the second scenario, they proved that $k$ instances of RSA public key pairs $\left(N_{i}, e_{i}\right)$ satisfying $e_{i} d_{i}-y \phi\left(N_{i}\right)=z_{i}$ for unknown integers $x_{i}, y, z_{i}$ where $x<N^{\delta}, y_{i}<N^{\delta},\left|z_{i}\right|<\frac{p_{i}-q_{i}}{3\left(p_{i}+q_{i}\right)} y_{i} N^{\frac{1}{4}}$ for 
$\delta=\frac{k(2 \alpha-1)}{2(k+1)}, N=\min \left\{N_{i}\right\}$ and $\min \left\{e_{i}\right\}=N^{\alpha}$. They used simultaneous Diophantine approximations and lattice basis reduction techniques and finally use the Coppersmith's method to compute prime factors $p_{i}$ and $q_{i}$ of RSA moduli $N_{i}$ in polynomial time.

Furthermore, Shehu and Ariffin (2017) also presented a polynomial time attack on $j$ instances of prime power RSA with modulus $N_{i}=p_{i}^{r} q_{i}$ using a good approximation of $\phi(N)$ in which they proved that for $j, r \geq 2$ and given public key pairs $\left(N_{i}, e_{i}\right)$ and $N=\min \left\{N_{i}\right\}$, then equation $e_{i} d-k_{i} \phi\left(N_{i}\right)=1$ can be satisfied only if the unknown integer $d<N^{\delta}$ and $j$ integers $k_{i}<N^{\delta}$ where $\delta=\frac{j-\gamma j}{j+1}$ for $0 \leq \gamma<1$, as reported in [14]. Also using equation $e_{i} d_{i}-k \phi\left(N_{i}\right)=1$, Shehu and Ariffin (2014) showed that $j$ prime power RSA modulus $N_{i}=p_{i}^{r} q_{i}$ can be simultaneously factored if the $j$ integers $d_{i}<N^{\delta}$ and integer $k<N^{\delta}, N=\min \left\{N_{i}\right\}$, and $\min \left\{e_{i}\right\}=N^{\beta}$ where $\delta=\frac{j(\beta-\gamma)}{j+1}$ for $\gamma<\beta<1$, [14].

The findings of this paper is reported in two parts. In the first part, we work on the first variant of prime power modulus with equation of the form ed $\equiv 1$ $\left(\bmod p^{r-1}(p-1)(q-1)\right)$ using continued fraction method. Firstly, we construct a lemma which gives approximation of $\phi(N)$ given by $\phi(N)>N-\left[2^{\frac{2 r+1}{r+1}} N^{\frac{r}{r+1}}\right\rceil$ and formulate a theorem which shows that if the secret exponent $d<\frac{1}{\sqrt{2}} \sqrt{N-2^{\frac{2 r+1}{r+1}} N^{\frac{r}{r+1}}}$, then one of the convergents $\frac{k}{d}$ can be found from the continued fraction expansion of $\frac{e}{N-\left[2^{\frac{2 r+1}{r+1}} N^{\frac{r}{r+1}}\right]}$ which leads to the factorization of prime power modulus $N=p^{r} q$ in polynomial time for $r \geq 2$. The paper also gives numerical example to justify how Theorem 3.2 works.

The second part of this paper presents cryptanalysis attacks of factoring $t$ instances of prime power moduli $N_{s}=p_{s}^{r} q_{s}$ in which we show that the moduli can be factored simultaneously using simultaneous Diophantine approximations and lattice basis reduction techniques. We present four new attacks using system of equations of the form $e_{s} d-k_{s} \phi\left(N_{s}\right)=1, e_{s} d_{s}-k \phi\left(N_{s}\right)=1, e_{s} d-k_{s} \phi\left(N_{s}\right)=z_{s}$ and $e_{s} d_{s}-k \phi\left(N_{s}\right)=z_{s}$ for $s=1, \ldots, t$, for $r \geq 2$ where the parameters $d, d_{s}$, $k, k_{s}$, and $z_{s}$ are unknown positive integers. In all the presented attacks, we have improved decryption exponent bound of some reported attacks.

The rest of the paper is organize as follows. In Section 2, we present review of some basic definitions of the terms used such as continued fraction, lattice basis reduction and some theorems that are related to our attacks. In Section 3, we present the proofs of our main results with lemma and theorems and their respective numerical examples and finally in Section 4 , we conclude the paper.

\section{PRELIMINARIES}

In this section, we present some basic definitions on continued fraction, lattice basis reduction and some theorems on continued fraction, LLL and simultaneous Diophantine approximations.

Definition 2.1. ( Continued fraction) The continued fraction of a real number $x$ is an expression of the form 


$$
x=a_{0}+\frac{1}{a_{1}+\frac{1}{a_{2}+\frac{1}{a_{3}+\ldots}}}
$$

This expression is often used in the form $x=\left[a_{0}, a_{1}, a_{2}, \ldots,\right]$. Any rational number $\frac{a}{b}$ can be expressed as a finite continued fraction $x=\left[a_{0}, a_{1}, a_{2} \ldots a_{m}\right]$. For $i \geq 0$, we define the $i^{\text {th }}$ convergent of the continued fraction $\left[a_{0}, a_{1}, a_{2}, \ldots,\right]$ to be $\left[a_{0}, a_{1}, a_{2}, \ldots, a_{i}\right]$. Each convergent is a rational number.

Definition 2.2. Let $\overrightarrow{b_{1}}, \cdots \overrightarrow{b_{m}} \in \mathcal{R}^{n}$. The vectors $b_{i}^{\prime} s$ are said to be linearly dependent if there exist $x_{1}, \ldots, x_{m} \in R$, which are not all zero and such that

$$
\sum_{i}^{m} x_{i} b_{i}=0 .
$$

Otherwise, they are said to be linearly independent.

Definition 2.3. (Lenstra et al. 1982) Let $n$ be a positive integer. A subset $\mathcal{L}$ of an $n$-dimensional real vector space $\mathcal{R}^{n}$ is called a lattice if there exists a basis $b_{1} \ldots b_{n}$ on $\mathcal{R}^{n}$ suchthat $\mathcal{L}=\sum_{i=1}^{n} \mathcal{Z} b_{i}=\sum_{i=1}^{n} r_{i} b_{i}: r_{i} \in \mathcal{Z}, 1 \leq i \leq n$.

In this situation, we say that $b_{1} \cdots b_{n}$ are basis for $\mathcal{L}$ or that they span $\mathcal{L},[15$.

Definition 2.4. (LLL Reduction) [16] Let $\mathcal{B}=\left\langle b_{1} \cdots b_{n}\right\rangle$ be a basis for a lattice $\mathcal{L}$ and let $B^{*}=\left\langle b_{1}^{*} \cdots b_{n}^{*}\right\rangle$ be the associated Gram-Schmidt orthogonal basis. Let

$$
\mu_{i, j}=\frac{\left\langle b_{i}, b_{j}^{*}\right\rangle}{\left\langle b_{j}^{*}, b_{j}^{*}\right\rangle} \text { for } 1 \leq j<i .
$$

The basis $\mathcal{B}$ is said to be $L L L$ reduce if it satisfies the following two conditions:

(1) $\mu_{i, j} \leq \frac{1}{2}$, for $1 \leq j<i \leq n$.

(2) $\frac{3}{4}\left\|b_{i-1}^{*}\right\|^{2} \leq\left\|b_{i}^{*}+\mu_{i, i-1} b_{i-1}^{*}\right\|^{2}$ for $1 \leq i \leq n$. Equivalently, it can be written as

$$
\left\|b_{i}^{*}\right\|^{2} \geq\left(\frac{3}{4}-\mu_{i, i-1}^{2}\right)\left\|b_{i-1}^{*}\right\|^{2} .
$$

Theorem 2.1. If $\frac{p_{1}}{q_{1}}, \frac{p_{2}}{q_{2}}, \ldots, \frac{p_{k}}{q_{k}}, \ldots$ are convergents of the simple continued fraction $\left[a_{1}, a_{2}, \ldots, a_{k}, \ldots\right]$, then the numerators and denominators of these convergents satisfy the following recursive relations:

$$
\begin{gathered}
p_{1}=a_{1}, p_{2}=a_{2} a_{1}+1, p_{k}=a_{k} p_{k-1}+p_{k-2}, \\
q_{1}=1, q_{2}=a_{2}, \quad q_{k}=a_{k} q_{k-1}+q_{k-2},
\end{gathered}
$$

for $k \geq 3,[17$.

Theorem 2.2. Let $\alpha$ be an arbitrary real number. If the rational number $\frac{p}{q}$ satisfies

$$
\left|\alpha-\frac{p}{q}\right|<\frac{1}{2 q^{2}}
$$

then $\frac{p}{q}$ must be a convergent of $\alpha$.

Theorem 2.3. Let $\mathcal{L}$ be a lattice basis of dimension $n$ having a basis $v_{1} \cdots v_{n}$. The $L L L$ algorithm produces a reduced basis $b_{1} \cdots b_{n}$ satisfying the following condition

$$
\left\|b_{1}\right\| \leq\left\|b_{2}\right\| \leq \cdots \leq\left\|b_{j}\right\| \leq 2^{\frac{n(n-1)}{4(n+1-j)}} \operatorname{det}(\mathcal{L})^{\frac{1}{n+1-j}}
$$

for all $1 \leq j \leq n,[15$. 
Theorem 2.4. (Simultaneous Diophantine Approximations, 13]) Given any rational numbers of the form $\alpha_{1}, \ldots, \alpha_{n}$ and $0<\varepsilon<1$, there is a polynomial time algorithm to compute integers $p_{1}, \ldots, p_{n}$ and a positive integer $q$ such that

$$
\max _{i}\left|q \alpha_{i}-p_{i}\right|<\varepsilon \text { and } q \leq 2^{\frac{n(n-3)}{4}} \cdot 3^{n} \cdot \varepsilon^{-n} .
$$

\section{MAIN RESULTS}

This section has two parts. The first part reports short decryption exponent attack on prime power modulus $N=p^{r} q$ using continued fraction method which leads to the successful factorization of the modulus in polynomial time. In the second part of the paper, we present cryptanalysis attacks using simultaneous Diophantine approximations and lattice basis reduction methods in factoring $t$ prime power modulus $N_{s}=p_{s}^{r} q_{s}$ using system of equations of the form $e_{s} d-k_{s} \phi\left(N_{s}\right)=1$, $e_{s} d_{s}-k \phi\left(N_{s}\right)=1, e_{s} d-k_{s} \phi\left(N_{s}\right)=z_{s}$ and $e_{s} d_{s}-k \phi\left(N_{s}\right)=z_{s}$ for $s=1, \ldots, t$, for $r \geq 2$ where parameters $d, d_{s}, k, k_{s}$ and $z_{s}$ are unknown positive integers.

\subsection{Cryptanalytic Attack Through Analyzing Approximation of $\phi(N)$} given by $N-\left\lceil 2^{\frac{2 r+1}{r+1}} N^{\frac{r}{r+1}}\right\rceil$.

This section presents a lemma which shows that if $q<p<2 q$ and the prime power modulus $N=p^{r} q$, then $\phi(N)>N-\left[2^{\frac{2 r+1}{r+1}} N^{\frac{r}{r+1}}\right]$ where $p$ and $q$ are distinct prime factors of the modulus $N=p^{r} q$, for $r \geq 2$. The section also proves a theorem which shows that the prime factors $p$ and $q$ can be recovered efficiently if $d<\frac{1}{\sqrt{2}} \sqrt{N-2^{\frac{2 r+1}{r+1}} N^{\frac{r}{r+1}}}$.

Lemma 3.1. Let $p$ and $q$ be prime numbers where $p<q<2 p$ and $N=p^{r} q$ for $r \geq 2$. If $e<\phi(N)$ and $N^{\frac{1}{r+1}}<p<2^{\frac{1}{r+1}} N^{\frac{1}{r+1}}$, then $\phi(N)>N-\left\lceil 2^{\frac{2 r+1}{r+1}} N^{\frac{r}{r+1}}\right\rceil$.

Proof. Let $N=p^{r} q$ and the condition $q<p<2 p$ holds, then multiplying by $p^{r}$ yields $N^{\frac{1}{r+1}}<p<2^{\frac{1}{r+1}} N^{\frac{1}{r+1}}$.

Using $\phi(N)=p^{r-1}(p-1)(q-1)$, gives the following

$$
\begin{aligned}
\phi(N) & =p^{r-1}(p-1)(q-1) \\
& =N-p^{r}-p^{r-1} q+p^{r-1} \\
N-\phi(N) & =p^{r}+p^{r-1} q-p^{r-1} \\
& <p^{r}+p^{r-1} q .
\end{aligned}
$$

Since $q<p$ and $p<2^{\frac{1}{r+1}} N^{\frac{1}{r+1}}$, then we have

$$
\begin{aligned}
N-\phi(N) & <2 p^{r} \\
& <2^{\frac{2 r+1}{r+1}} N^{\frac{r}{r+1}} .
\end{aligned}
$$

Hence $\phi(N)>N-2^{\frac{2 r+1}{r+1}} N^{\frac{r}{r+1}}$.

Theorem 3.2. Let $p$ and $q$ be prime numbers satisfying $p<q<2 p$ and let $N=p^{r} q$ be prime power modulus where $(N, e)$ and $(N, d)$ are public and private keys pairs respectively with $e<\phi(N)$. If the decryption exponent $d<\frac{1}{\sqrt{2}} \sqrt{N-2^{\frac{2 r+1}{r+1}} N^{\frac{r}{r+1}}}$, then one of the convergents $\frac{k}{d}$ can be found from the continued fraction expansion of 
$\frac{e}{N-\left[2^{\frac{2 r+1}{r+1}} N^{\frac{r}{r+1}}\right\rceil}$ which leads to the factorization of prime power modulus $N=p^{r} q$ for $r \geq 2$ in polynomial time.

Proof. Observe

$$
\begin{aligned}
\frac{e d-k \phi(N)}{d \phi(N)} & =\frac{e}{\phi(N)}-\frac{k}{d} \\
& =\frac{1}{d \phi(N)} \\
& >0
\end{aligned}
$$

Taking $N-\left\lceil 2^{\frac{2 r+1}{r+1}} N^{\frac{r}{r+1}}\right\rceil$ from Lemma 3.1 as approximation of $\phi(N)$ yields:

$$
\begin{aligned}
\frac{e}{\phi(N)}-\frac{k}{d} & =\frac{e}{N-\left\lceil 2^{\frac{2 r+1}{r+1}} N^{\frac{r}{r+1}}\right\rceil}-\frac{e}{\phi(N)}+\frac{e}{\phi(N)}-\frac{k}{d} \\
& =\frac{e\left(N-\phi(N)-\left\lceil 2^{\frac{2 r+1}{r+1}} N^{\frac{r}{r+1}}\right\rceil\right)}{\phi(N)\left(N-\left\lceil 2^{\frac{2 r+1}{r+1}} N^{\frac{r}{r+1}}\right\rceil\right)}+\frac{e}{\phi(N)}-\frac{k}{d}
\end{aligned}
$$

Since $N-\phi(N)<\left\lceil 2^{\frac{2 r+1}{r+1}} N^{\frac{r}{r+1}}\right\rceil$, let $\frac{e\left(N-\phi(N)-\left\lceil 2^{\frac{2 r+1}{r+1}} N^{\frac{r}{r+1}}\right\rceil\right)}{\phi(N)\left(N-\left\lceil 2^{\frac{2 r+1}{r+1}} N^{\frac{r}{r+1}}\right\rceil\right)}=T<0$, then

$$
\begin{aligned}
& =\frac{e}{\phi(N)}-\frac{k}{d}-T \\
& <\frac{e}{\phi(N)}-\frac{k}{d} \\
& =\frac{1}{d \phi(N)} \\
& <\frac{1}{\phi(N)} .
\end{aligned}
$$

It was shown from Lemma 3.1 that $\phi(N)>N-2^{\frac{2 r+1}{r+1}} N^{\frac{r}{r+1}}$, this implies

$$
\frac{1}{\phi(N)}<\frac{1}{N-2^{\frac{2 r+1}{r+1}} N^{\frac{r}{r+1}}}
$$

Since $d<\frac{1}{\sqrt{2}} \sqrt{N-2^{\frac{2 r+1}{r+1}} N^{\frac{r}{r+1}}}$, then

$$
\frac{1}{N-2^{\frac{2 r+1}{r+1}} N^{\frac{r}{r+1}}}<\frac{1}{2 d^{2}} \text {. }
$$

Hence,

$$
\left|\frac{e}{N-2^{\frac{2 r+1}{r+1}} N^{\frac{r}{r+1}}}-\frac{k}{d}\right|<\frac{1}{2 d^{2}} .
$$

This shows that Theorem 3.2 produces $\frac{k}{d}$ as one of the convergent of the continued fraction expansion of $\frac{e}{N-2^{\frac{2 r+1}{r+1}} N^{\frac{r}{r+1}}}$. This terminates the proof.

The section also outlines below the algorithm to be followed in factoring the prime power modulus $N=p^{r} q$ for $r \geq 2$. 


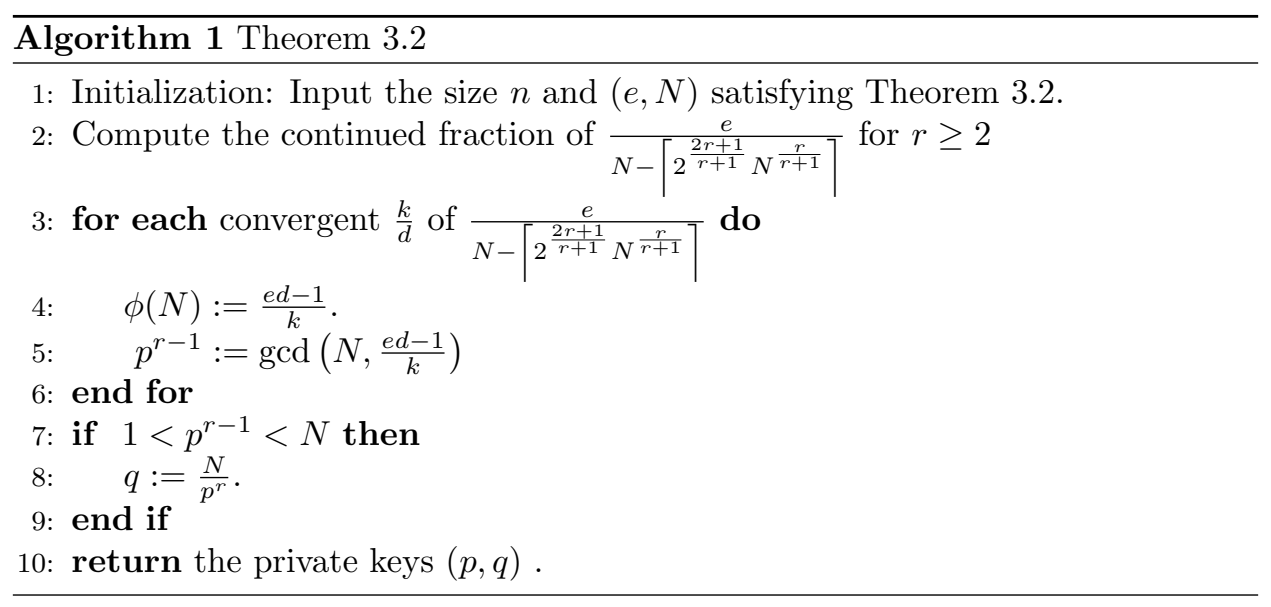

Example 3.1. This example gives an illustration of how Theorem 3.2 works on prime power modulus $N=p^{r} q$ for $r=3$.

Let $N=6467824680967991485093968594984906698452846918126619877544795476$ 4512899975949030092389431143550672950630682676159477346727505541 7769195369573715802340930197206294064847562258550047184856229657 624642567132668279698576503914916943223342223042619190115630551 $e=73911690643137255586285890252274214143777796475233050043697253070$ 5528100075149987700910725916512370037680964946694446080570622352 30891956543988833677211276168333624077420583009020578268295151250 34066183663278580767186726153429453492047380587411856008249.

Taking the continued fraction expansion of $\frac{e}{N-\left[2^{\frac{2 r+1}{r+1}} N^{\frac{r}{r+1}}\right]}$ for $r=3$, gives the following: $[0,87,1,1,32,1,95,1,13,1,13,1,7,2,6,2,6,2,1,2,2,4,7,580,1,22,5,3,30,1,1,3,3$, $1,14,12,5,2,26,2,3,2,1,1,1,1,9,1,16,4,1,2,1,1,4,5,1,1,1,32,1,76,13,1,2,1,14$, $1,1,22,1,5,1,40,1,5,2,2,3,1,1,4,273,3,1,40,3,15,1,3,1,10,36,1,43,1,3,2,1,1,1$, $4,2,2,3,3,2,3,1,2,1,10,1,10,1,1,1,1,9,1,1,5,1,4,2,1,9,10,1,6,8,2,4,4,6,1, \ldots$,$] .$ Then the convergent $\frac{k}{d}$ is found from the continued fraction expansion of $\frac{e}{N-\left\lceil 2^{\frac{2 r+1}{r+1}} N^{\frac{r}{r+1}}\right\rceil}$ as

$$
\frac{k}{d}=\frac{5283691555749297587344711786335}{462362453808524086451896135480609} .
$$

From Algorithm 1, we compute $\phi(N)=\frac{e d-1}{k}$ as follows:

$$
\begin{aligned}
\phi(N)= & 64678246809679914850939685949849066984528469181266198775447954734769 \\
& 19313372348183843208998399569436036670863930678884295028399128045789 \\
& 530812942035589777693967687689231460940380248219361255263103210104370 \\
& 18161613390372921479673079645463655977965218274984 .
\end{aligned}
$$

Finally, from Algorithm 1 the following computations reveal the prime factors $p$ and $q$ of the prime power modulus $N=p^{r} q$ : 


$$
\begin{aligned}
p^{r-1} & =\operatorname{gcd}(N, \phi(N)) \\
p & =5684119572206954830995467120947108108574615439214643985219161027 \\
q & =\frac{N}{p^{3}} \\
q & =3521831905037505963424663411629941658417389143836791215207878197 .
\end{aligned}
$$

From our result, one can observe that, this work yields $d \approx N^{0.1281}$ which is greater than Shehu-Ariffin's bound $d \approx N^{0.102}$, as reported in [14.

3.2. Cryptanalysis Attacks on $t$ Prime Power With Moduli $N_{s}=p_{s}^{r} q_{s}$ Using $N-2^{\frac{2 r+1}{r+1}} N^{\frac{r}{r+1}}$ as Approximation of $\phi(N)$.

This section presents four successful cryptanalysis attacks of factoring $t$ prime power with moduli $N_{s}=p_{s}^{r} q_{s}$ using systems of equations $e_{s} d-k_{s} \phi\left(N_{s}\right)=1$, $e_{s} d_{s}-k \phi\left(N_{s}\right)=1, e_{s} d-k_{s} \phi\left(N_{s}\right)=z_{s}$ and $e_{s} d_{s}-k \phi\left(N_{s}\right)=z_{s}$ where the parameter $\phi(N)=N-\left(p^{r}+p^{r-1} q-p^{r-1}\right)$ for $r \geq 2$ and $s=1, \ldots, t$.

3.2.1. The Attack on $t$ Prime Power Moduli $N_{s}=p_{s}^{r} q_{s}$ Satisfying System of Equation $e_{s} d-k_{s} \phi\left(N_{s}\right)=1$.

Taking $t \geq 2$, let $N_{s}=p_{s}^{r} q_{s}$, for $s=1, \ldots, t$ and $r \geq 2$. The attack works for $t$ instances of the public key tuple $\left(N_{s}, e_{s}\right)$ when there exists an integer $d$ and $t$ integers $k_{s}$ satisfying equation $e_{s} d-k_{s} \phi\left(N_{s}\right)=1$. It shows that $t$ prime factors $p_{s}$ and $q_{s}$ of $t$ prime power with moduli $N_{s}=p_{s}^{r} q_{s}$ for $s=1, \ldots, t, r \geq 2$ can be found efficiently for $N=\max \left\{N_{s}\right\}$ and $d<N^{\varrho}, k_{s}<N^{\varrho}$, for all $\varrho=\frac{t(1-\beta)}{t+1}$ for $0<\beta<1$. In this case, the instances $\left(N_{s}, e_{s}\right)$ shared common decryption exponent $d$.

Theorem 3.3. Let $N_{s}=p_{s}^{r} q_{s}$ be prime power moduli for $r \geq 2, s=1, \cdots, t$ and $t \geq 2$. Let $\left(N_{s}, e_{s}\right)$ be public key pair and $\left(d, N_{s}\right)$ be private key pair with condition $e_{s}<\phi\left(N_{s}\right)$ and the relation $e_{s} d \equiv 1 \bmod \phi\left(N_{s}\right)$ is satisfied. Let $N=\max \left\{N_{s}\right\}$. If there exists positive integers $d<N^{\varrho}, k_{s}<N^{\varrho}$, for all $\varrho=\frac{t(1-\beta)}{t+1}$ such that equation $e_{s} d-k_{s} \phi\left(N_{s}\right)=1$ holds, for $0<\beta<1$, then $t$ prime power moduli $N_{s}$ can successfully be factored in polynomial time for $\frac{1}{4} \leq \varrho \leq \frac{1}{2}$ and $0<\beta<1$.

Proof. For $r, t \geq 2$ where $N_{s}=p_{s}^{r} q_{s}$ is prime power moduli. Suppose that $N=$ $\max \left\{N_{s}\right\}$ and $k_{s}<N^{\varrho}$ for $s=1, \ldots, t$. Then equation $e_{s} d-k_{s} \phi\left(N_{s}\right)=1$ can be rewritten as follows:

$$
e_{s} d-k_{s}\left(N_{s}-\left(N_{s}-\phi(N)_{s}\right)\right)=1
$$

Let $\triangle=2^{\frac{2 r+1}{r+1}} N^{\frac{r}{r+1}}$

$$
\begin{gathered}
e_{s} d-k_{s}\left(N_{s}-\triangle+\triangle-\left(N_{s}-\phi\left(N_{s}\right)\right)\right)=1 \\
\left|\frac{e_{s}}{N-\triangle} d-k_{s}\right|=\frac{\left|1-k_{s}\left(N_{s}-\phi\left(N_{s}\right)-\triangle\right)\right|}{N_{s}-\triangle} .
\end{gathered}
$$

Since $N=\max \left\{N_{s}\right\}$ and $k_{s}<N_{s}^{\varrho}, d<N^{\varrho}$ be positive integers. Observe

$$
\left|N_{s}-\phi\left(N_{s}\right)-\triangle\right|<N_{s}^{\beta}<N^{\beta}
$$


for $\beta \in(0,1)$ and

$$
N_{s}-\triangle>\frac{1}{r+2} N
$$

then plugging into equation 3.1 gives

$$
\begin{aligned}
\left|\frac{1-k_{s}\left(N_{s}-\phi\left(N_{s}\right)-\triangle\right)}{N_{s}-\triangle}\right| & <\frac{\left|1+k_{s}\left(N_{s}-\phi\left(N_{s}\right)-\triangle\right)\right|}{N_{s}-\triangle} \\
& <\frac{1+N^{\varrho}\left(N^{\beta}\right)}{\frac{1}{r+2} N} \\
& =\frac{r+2\left(1+N^{\varrho+\beta}\right)}{N} \\
& <\sqrt{2 r} N^{\varrho+\beta-1} .
\end{aligned}
$$

Then, it follows that

$$
\left|\frac{e_{s}}{N_{s}-\triangle} d-k_{s}\right|<\sqrt{2 r} N^{\varrho+\beta-1} .
$$

We proceed to show the existence of integer $d$ and $t$ integers $k_{s}$. Let $\varepsilon=\sqrt{2 r} N^{\varrho+\beta-1}$, with $\varrho=\frac{t(1-\beta)}{t+1}$. Then it gives

$$
N^{\varrho} \varepsilon^{t}=N^{\varrho}\left(\sqrt{2 r} N^{\varrho+\beta-1}\right)^{t}=(2 r)^{\frac{t}{2}} N^{\varrho+\varrho t+\beta t-t}=(2 r)^{\frac{t}{2}} .
$$

Following Theorem 2.4 $(2 r)^{\frac{t}{2}}<2^{\frac{t(t-3)}{4}} \cdot 3^{t}$ for $t, r \geq 3$, then $N \varrho \varepsilon^{t}<2^{\frac{t(t-3)}{4}} \cdot 3^{t}$. It follows that since $d<N^{\varrho}$ then $d<2^{\frac{t(t-3)}{4}} \cdot 3^{t} \cdot \varepsilon^{-t}$ for $s=1, \cdots, t$, yields

$$
\left|\frac{e_{s}}{N_{s}-\triangle} d-k_{s}\right|<\varepsilon \text {. }
$$

This clearly satisfies the conditions of Theorem 2.4, and proceeds to reveal the private key $d$ and $t$ integers $k_{s}$ for $s=1, \ldots, t$. Next, from $e_{s} d-k_{s} \phi\left(N_{s}\right)=1$ we perform the following computations:

$$
\begin{aligned}
\phi\left(N_{s}\right) & =\frac{e_{s} d-1}{k_{s}} \\
p_{s}^{r-1} & =\operatorname{gcd}\left(\phi\left(N_{s}\right), N_{s}\right) \\
q_{s} & =\frac{N_{s}}{p_{s}^{r}} .
\end{aligned}
$$

Finally, the prime factors $p_{s}$ and $q_{s}$ can be revealed which leads to the factorization of $t$ prime power moduli $N_{s}$ for $s=1, \cdots, t$ in polynomial time.

Let

$$
\begin{aligned}
& X_{1}=\frac{e_{1}}{N_{1}-2^{\frac{2 r+1}{r+1}} N_{1}^{\frac{r}{r+1}}}, \\
& X_{2}=\frac{e_{2}}{N_{2}-2^{\frac{2 r+1}{r+1}} N_{2}^{\frac{r}{r+1}}}, \\
& X_{3}=\frac{e_{3}}{N_{3}-2^{\frac{2 r+1}{r+1}} N_{3}^{\frac{r}{r+1}}} .
\end{aligned}
$$

Define,

$$
T=\left[3^{t+1} \times 2^{\frac{(t+1)(t-4)}{4}} \times \varepsilon^{-t-1}\right] .
$$


Consider the lattice $\mathcal{L}$ spanned by the matrix,

$$
M=\left[\begin{array}{cccc}
1 & -\left[T\left(X_{1}\right)\right] & -\left[T\left(X_{2}\right)\right] & -\left[T \times X_{3}\right] \\
0 & T & 0 & 0 \\
0 & 0 & T & 0 \\
0 & 0 & 0 & T
\end{array}\right]
$$

Taking $r \geq 2$, the matrix $M$ can be used in computing the reduced basis after applying the LLL algorithm.

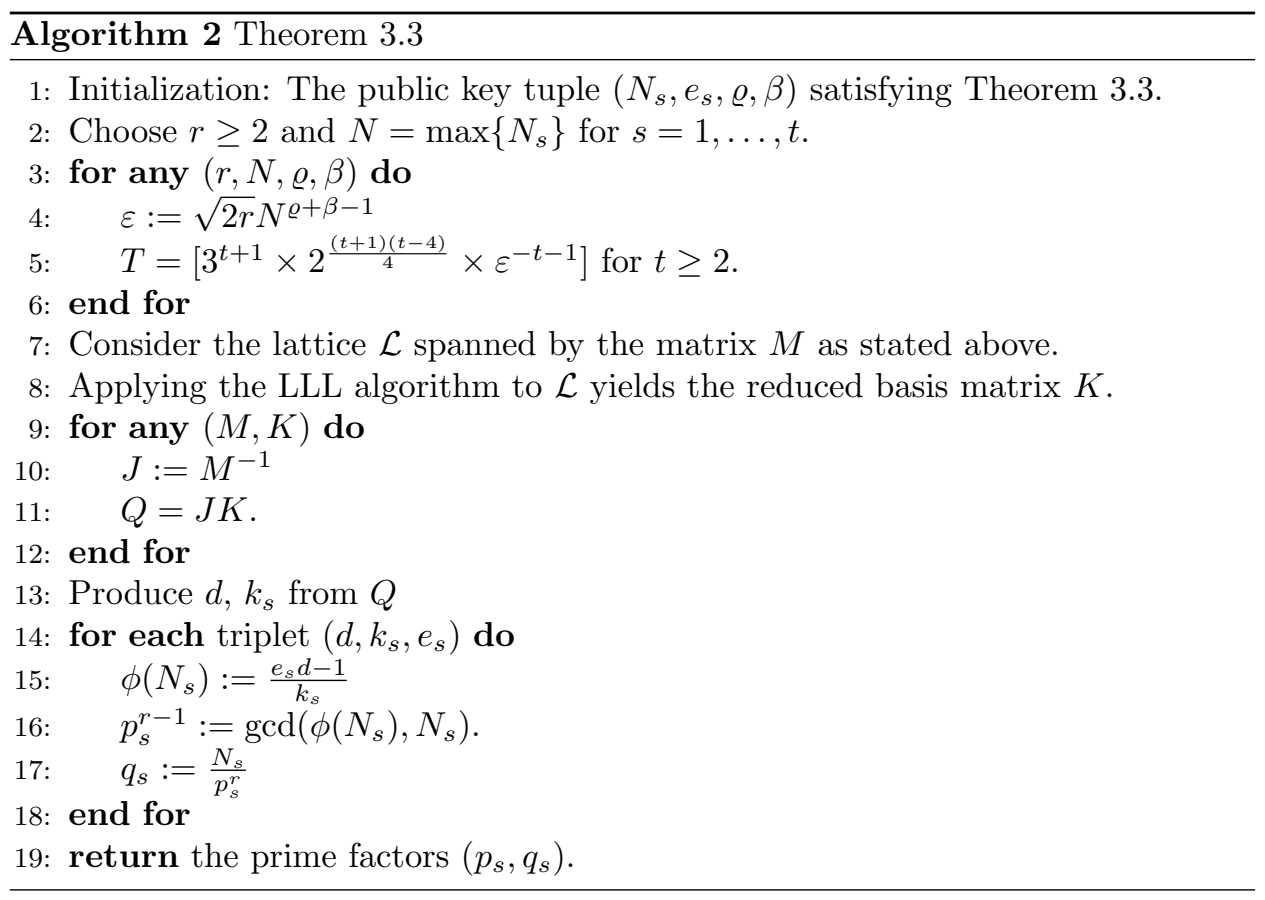

Example 3.2. This example gives an illustration of how Theorem 3.3 works on 3 prime power moduli also their corresponding public exponents:

Let $N_{1}=563382281374803858489382903716443474446580306437566005728878179$ 267676092551665191432331661132041057581935108036853538725342976031062566 064493977301796320064579931954653

$N_{2}=1107801608689388607908020314275395456891637713924780000534249617$ 140001335413025467235568243203922733387749142234285602245999359726144181 863789668190983522850626483669023

$N_{3}=9654013301685016055400506098375594830137130427693056264836899674$ 069160933238013628743471068194753666107834756424555627523960922969439392 11051267861006991380940921618139

$e_{1}=16764458147751748810556293131530021884042990680920812662235184222$ 412584386285442254876550471043222816261798853928202440252160827176635480 


\section{1}

$e_{2}=46315246118060854234591247117247522105087920085609401490448821248$ 150485833350212292332828566638083188341508684445351764219211533501687950 3086688812611607958721881159677

$e_{3}=41767933005026254973096790277305670676920459090792054979090531316$ 476588371879849877872939920171606752882950570359186955894106589500439562 792307841833725917421212937261

Observe that $N=\max \left\{N_{1}, N_{2}, N_{3}\right\}$

$N=11078016086893886079080203142753954568916377139247800005342496171$ 400013354130254672355682432039227333877491422342856022459993597261441818 63789668190983522850626483669023.

Using Algorithm 2 for $t=3 r=3$ and $\beta=0.75$ gives $\varrho=\frac{t(1-\beta)}{t+1}=0.1875$ and $\varepsilon=\sqrt{2 r} N^{\varrho+\beta-1}=9.111089161 \times 10^{-39}$.

Applying Theorem 2.4 and using Algorithm 2 for $n=t=3$, we compute

$$
T=\left[3^{t+1} \cdot 2^{\frac{(t+1)(t-4)}{4}} \cdot \varepsilon^{-t-1}\right.
$$

$T=1154165320000000000000000000000000000000000$

Consider the lattice $\mathcal{L}$ spanned by the matrix,

$$
M=\left[\begin{array}{cccc}
1 & -\left[T\left(X_{1}\right)\right] & -\left[T\left(X_{2}\right)\right] & -\left[T \times X_{3}\right] \\
0 & T & 0 & 0 \\
0 & 0 & T & 0 \\
0 & 0 & 0 & T
\end{array}\right]
$$

Therefore, by applying the LLL algorithm to $\mathcal{L}$, it yields the reduced basis with the following matrix

$$
K=\left[\begin{array}{llll}
A_{11} & A_{12} & A_{13} & A_{14} \\
B_{11} & B_{12} & B_{13} & B_{14} \\
C_{11} & C_{12} & C_{13} & C_{14} \\
D_{11} & D_{12} & D_{13} & D_{14}
\end{array}\right]
$$

where

$A_{11}=7770294469564621426285729048713, A_{12}=-11866076834029234002241236377582$

$A_{13}=-32633775130445983893911306068132, A_{14}=-3328082490144200863069292352913$

$B_{11}=18663562246576439716517789824933, B_{12}=15048857017191042713184735691338$

$B_{13}=10286010869239647472878709783788, B_{14}=18549844424135999309545228522867$

$C_{11}=-17095263517456624755229311937397, C_{12}=-1750544855365507798774316440042$

$C_{13}=-3457459628216362153472612722092, C_{14}=25713796499666717792079771007197$

$D_{11}=-11796372669551527880579978498483, D_{12}=49475532903151632120923767998362$

$D_{13}=-3506878085541730219207678001588, D_{14}=2579429578946966788750278220683$. 
Next, from Algorithm 2, we compute $Q=K J$,

$$
Q=\left[\begin{array}{llll}
E_{11} & E_{12} & E_{13} & E_{14} \\
F_{21} & F_{22} & F_{23} & F_{24} \\
G_{31} & G_{32} & G_{33} & G_{34} \\
H_{41} & H_{24} & H_{43} & H_{44}
\end{array}\right]
$$

where

$E_{11}=7770294469564621426285729048713, E_{12}=2312191574659429845702482436055$

$E_{13}=3248624103312694525051599010754, E_{14}=336180538281891705775592037701$

$F_{21}=18663562246576439716517789824933, \quad F_{22}=5553680307573184886408327739349$

$F_{23}=7802908680667061550845174371910, F_{24}=807476013539447921932253556384$

$G_{31}=-17095263517456624755229311937397, G_{32}=-5087004672277331272333963880373$

$G_{33}=-7147230434164420892985948873697, G_{34}=-739623821707154665925571172837$

$H_{41}=-11796372669551527880579978498483, H_{42}=-3510223918142974267040005409244$

$H_{43}=-4931856924607830680600230836138, H_{44}=510368162925726525039096348715$.

From the second row of the matrix $Q$, it yields the values for $d, k_{1}, k_{2}$ and $k_{3}$ as follows:

$d=18663562246576439716517789824933, \quad k_{1}=5553680307573184886408327739349$

$k_{2}=7802908680667061550845174371910, k_{3}=807476013539447921932253556384$.

Using Algorithm 2, $\phi\left(N_{s}\right)=\frac{e_{s} d-1}{k_{s}}$ for $s=1,2,3$ can be computed as follows,

$\phi\left(N_{1}\right)=5633822813748038584893829037164434744465782155701645149991994$ 356443759551568355864223530483724579125013595134403585635257661391033125 76827433249968405344191335088982588

$\phi\left(N_{2}\right)=1107801608689388607908020314275395456891635042012854891240545$ 862148617356725153462170062810236431892835802535487477427440668918578671 114561643302523968636342406392082904

$\phi\left(N_{3}\right)=9654013301685016055400506098375594830137106311177123492452313$ 119418214171537111121456291483851298457821141362575639293601436535938050 40382205965865042430321233447659168 .

Next, from Algorithm $2 p_{s}^{r-1}$ for $s=1,2,3$ and $r=3$ can be computed as follows,

$$
\begin{aligned}
& p_{1}=1172087672819698576140295693798879515111959 \\
& p_{2}=1205801981963990013436312155116150241125443 \\
& p_{3}=1165539406118780488715861651907300862321907 .
\end{aligned}
$$

Finally, from Algorithm 2 $q_{s}$ for $s=1,2,3$ can be computed as follows,

$$
\begin{aligned}
& q_{1}=349883038156174349555037833667162924726907 \\
& q_{2}=631879129745772702497880264093194679122189 \\
& q_{3}=609715181679983501366253856456455327579473 .
\end{aligned}
$$


This shows the factorization of 3 prime power moduli $N_{s}=p_{s}^{r} q_{s}$ for $s=1,2,3$ and $r=3$ in polynomial time. One can also observe that, our work yield $d \approx$ $N^{0.18608}$ which is greater than $d \approx N^{0.1857}$, as reported in [14]. This shows that Shehu and Ariffin's attack can not yield the factorization of $t$ prime power moduli in our case.

3.2.2. The Attack on $t$ Prime Power Moduli $N_{s}=p_{s}^{r} q_{s}$ Satisfying System of Equation $e_{s} d_{s}-k \phi\left(N_{s}\right)=1$.

This section considers second case in which $t$ prime power moduli satisfies equations of the form $e_{s} d_{s}-k \phi\left(N_{s}\right)=1$ for unknown positive integers $d_{s}$ and $k$ for $s=1, \ldots, t$. In this case, every pair of the instances $\left(N_{s}, e_{s}\right)$ has its own unique decryption exponent $d_{s}$.

Theorem 3.4. Let $N_{s}=p_{s}^{r} q_{s}$ be prime power moduli where $p_{s}$ and $q_{s}$ are prime numbers for $s=1, \ldots, t, r, t \geq 2$. Let $\left(e_{s}, N_{s}\right)$ be public key pair and $\left(d_{s}, N_{s}\right)$ be private key pair with $e_{s}<\phi\left(N_{s}\right)$ and the relation $e_{s} d_{s} \equiv 1 \bmod \phi\left(N_{s}\right)$ is satisfied. Let $e=\min \left\{e_{s}\right\}=N^{\alpha}$ be public exponent. If there exists $t$ integers $d_{s}<N^{\varrho}$ and integer $k<N^{\varrho}$, for all $\varrho=\frac{t(\alpha-\beta)}{t+1}$ such that $e_{s} d_{s}-k \phi\left(N_{s}\right)=1$ holds, then prime factors $p_{s}$ and $q_{s}$ of $t$ prime power moduli $N_{s}$ can be successfully recovered in polynomial time for $0<\varrho \leq \frac{1}{2}, 0<\beta<1$ and $\beta<\alpha<1$.

Proof. For $r, t \geq 2$ and $N_{s}=p_{s}^{r} q_{s}$, be $t$ prime power moduli $e=\min \left\{e_{s}\right\}=N^{\alpha}$ be public exponent for $s=1, \ldots t$ and suppose that $d_{s}<N^{\varrho}$. Then equation $e_{s} d_{s}-k \phi\left(N_{s}\right)=1$ can be transformed into

$$
e_{s} d_{s}-k\left(N_{s}-\left(N_{s}-\phi\left(N_{s}\right)\right)\right)=1
$$

Let $\triangle=2^{\frac{2 r+1}{r+1}} N_{s}^{\frac{r}{r+1}}$

$$
\begin{aligned}
e_{s} d_{s}-k\left(N_{s}-\triangle+\triangle-\left(N_{s}-\phi\left(N_{s}\right)\right)\right) & =1 \\
e_{s} d_{s}-k\left(N_{s}-\triangle\right) & =1-k\left(N_{s}-\phi\left(N_{s}\right)-\triangle\right) \\
\left|k \frac{\left(N_{s}-\triangle\right)}{e_{s}}-d_{s}\right| & =\frac{\left|1-k\left(N_{s}-\phi\left(N_{s}\right)-\triangle\right)\right|}{e_{s}} .
\end{aligned}
$$

Since $N=\max \left\{N_{s}\right\}$ and $d_{s}<N^{\varrho}, k<N^{\varrho}$ are positive integers. Observe

$$
N_{s}-\phi\left(N_{s}\right)-\triangle<N_{s}^{\beta}<N^{\beta}
$$

for $\beta \in(0,1)$. Since also $e=\min \left\{e_{s}\right\}=N^{\alpha}$, for $s=1, \cdots, t$ then it gives

$$
\begin{aligned}
\frac{\left|1-k\left(N_{s}-\phi\left(N_{s}\right)-\triangle\right)\right|}{e_{s}} & \leq \frac{\left|1+k\left(N_{s}-\phi\left(N_{s}\right)-\triangle\right)\right|}{e_{s}} \\
& <\frac{1+N^{\varrho}\left(N^{\beta}\right)}{N^{\alpha}} \\
& =\frac{1+N^{\varrho+\beta}}{N^{\alpha}} \\
& <\sqrt{r} N^{\varrho+\beta-\alpha} .
\end{aligned}
$$

Hence,

$$
\left|k \frac{\left(N_{s}-\triangle\right)}{e_{s}}-d_{s}\right|<\sqrt{r} N^{\varrho+\beta-\alpha}
$$


We proceed to show the existence of integer $k$ and $t$ integers $d_{s}$. Taking $\varepsilon=$ $\sqrt{r} N^{\varrho+\beta-\alpha}$ and $\varrho=\frac{t(\alpha-\beta)}{t+1}$. Then it gives

$$
N^{\varrho} \varepsilon^{t}=N^{\varrho}\left(\sqrt{r} N^{\varrho+\beta-\alpha}\right)^{t}=(\sqrt{r})^{t} N^{\varrho+\varrho t+\beta t-\alpha t}=r^{\frac{t}{2}} .
$$

Following Theorem 2.4 $r^{\frac{t}{2}}<2^{\frac{t(t-3)}{4}} \cdot 3^{t}$ for $r, t \geq 2$, then it gives $N^{\varrho} \varepsilon^{t}<2^{\frac{t(t-3)}{4}} \cdot 3^{t}$. It follows that if $k<N^{\varrho}$ then $k<2^{\frac{t(t-3)}{4}} \cdot 3^{t} \cdot \varepsilon^{-t}$ for $s=1, \ldots, t$, yields

$$
\left|k \frac{\left(N_{s}-\triangle\right)}{e_{s}}-d_{s}\right|<\varepsilon
$$

This clearly satisfies the conditions of Theorem 2.4 and proceeds to reveal the private keys $k$ and $t$ integers $d_{s}$ for $s=1, \ldots, t$. Next from $e_{s} d_{s}-k \phi\left(N_{s}\right)=1$ we make the following computations :

$$
\begin{aligned}
\phi\left(N_{s}\right) & =\frac{e_{s} d_{s}-1}{k} \\
p_{s}^{r-1} & =\operatorname{gcd}\left(\phi\left(N_{s}\right), N_{s}\right) \\
q_{s} & =\frac{N_{s}}{p_{s}^{r}} .
\end{aligned}
$$

Finally, the prime factors $p_{s}$ and $q_{s}$ can be revealed which lead to the factorization of $t$ prime power moduli $N_{s}$ for $s=1, \ldots, t$ and $r \geq 2$.

Let

$$
\begin{aligned}
& X_{1}=\frac{N_{1}-2^{\frac{2 r+1}{r+1}} N_{1}^{\frac{r}{r+1}}}{e_{1}} \\
& X_{2}=\frac{N_{2}-2^{\frac{2 r+1}{r+1}} N_{2}^{\frac{r}{r+1}}}{e_{2}} \\
& X_{3}=\frac{N_{3}-2^{\frac{2 r+1}{r+1}} N_{3}^{\frac{r}{r+1}}}{e_{3}} .
\end{aligned}
$$

Define,

$$
T=\left[3^{t+1} \times 2^{\frac{(t+1)(t-4)}{4}} \times \varepsilon^{-t-1}\right] .
$$

Consider the lattice $\mathcal{L}$ spanned by the matrix,

$$
M=\left[\begin{array}{cccc}
\mathbf{1} & -\left[T\left(X_{1}\right)\right] & -\left[T\left(X_{2}\right)\right] & -\left[T\left(X_{3}\right)\right] \\
0 & T & 0 & 0 \\
0 & 0 & T & 0 \\
0 & 0 & 0 & T
\end{array}\right]
$$

Taking $r \geq 2$, the matrix $M$ can be used in computing the reduced basis after applying the LLL algorithm 


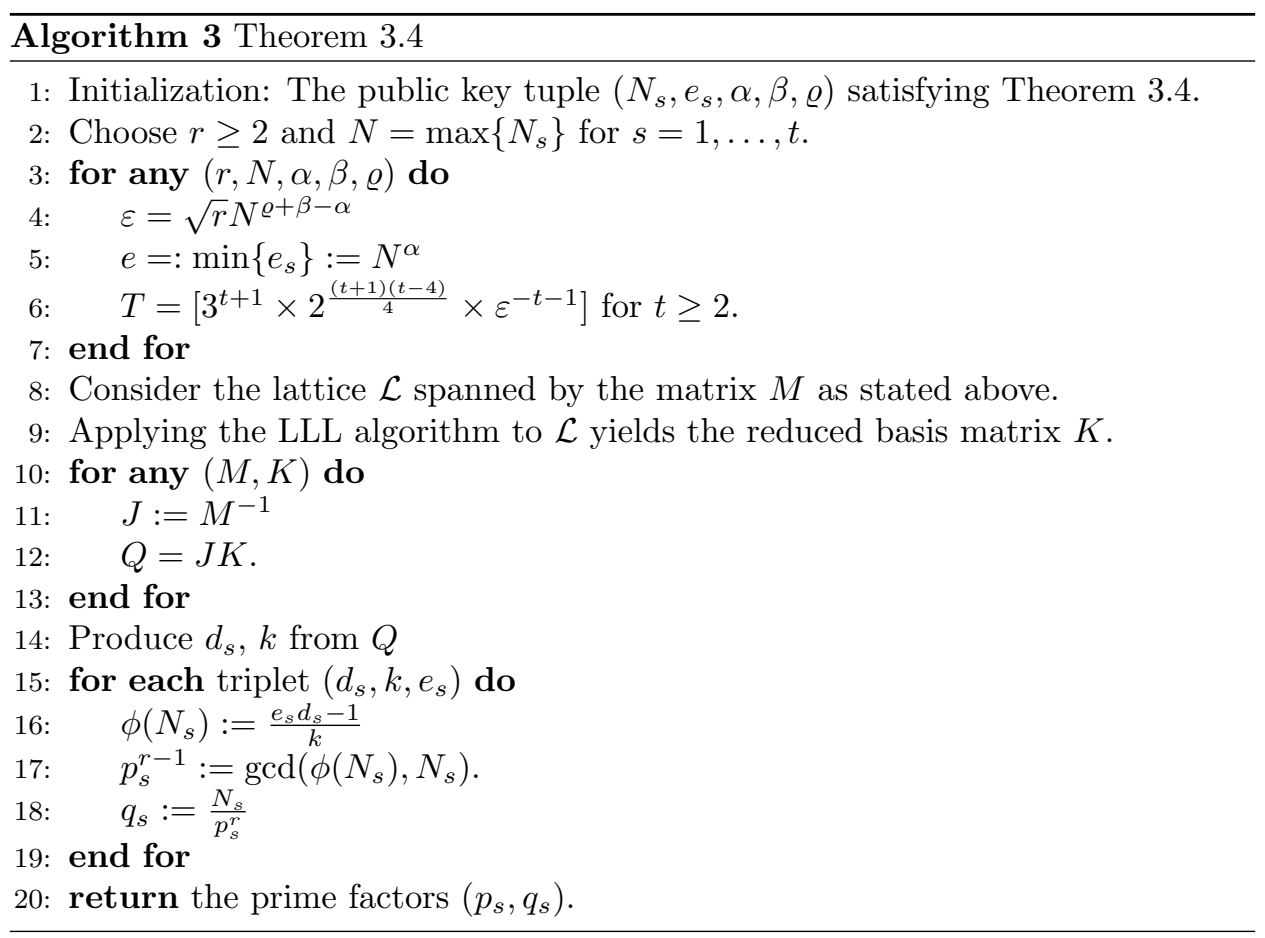

Example 3.3. This example gives an illustration of how Theorem 3.4 works on 3 prime power moduli and their corresponding public exponents:

$$
\begin{aligned}
N_{1}= & 23075243076701307224988768429391071883704004007139924828386666369151434 \\
& 45411611467379 \\
N_{2}= & 434991743050236060915996189147523264755865914949477613614468188806740 \\
& 952003913552583 \\
N_{3}= & 980914643623371382312729458097187264388503427621447777571718784533143 \\
& 406738292636683 \\
e_{1}= & 62904914881055994984178504976156821570002622680726423145736325680212 \\
& 648863937888039 \\
e_{2}= & 265035571511591897022174737291070924314658140619585199620247165205557 \\
& 379598308572799 \\
e_{3}= & 424302253973827276427319770823031080967261427337097513012296323366084 \\
& 850555293675453 .
\end{aligned}
$$

Observe

$$
\begin{aligned}
N=\max \left\{N_{1}, N_{2}, N_{3}\right\}= & 9809146436233713823127294580971872643885034276214477775717187 \\
& 84533143406738292636683 \\
e=\min \left\{e_{1}, e_{2}, e_{3}\right\}= & 62904914881055994984178504976156821570002622680726423145736 \\
& 325680212648863937888039
\end{aligned}
$$


with $e=\min \left\{e_{1}, e_{2}, e_{3}\right\}=N^{\alpha}$ for $\alpha=0.9857968390$. Taking $t=3, \beta=0.75$ it gives $\varrho=\frac{t(\alpha-\beta)}{t+1}=0.1768476292$ and $\varepsilon=0.00001937850804$.

Applying Theorem 2.4 and using Algorithm 3, we compute

$$
T=\left[3^{t+1} \cdot 2^{\frac{(t+1)(t-4)}{4}} \cdot \varepsilon^{-t-1}\right]=287192882900000000000 .
$$

Consider the lattice $\mathcal{L}$ spanned by the matrix,

$$
M=\left[\begin{array}{cccc}
\mathbf{1} & -\left[T\left(X_{1}\right)\right] & -\left[T\left(X_{2}\right)\right] & -\left[T\left(X_{3}\right)\right] \\
0 & T & 0 & 0 \\
0 & 0 & T & 0 \\
0 & 0 & 0 & T
\end{array}\right]
$$

Therefore, by applying the LLL algorithm to $\mathcal{L}$, it yields the reduced basis with the following matrix

$$
K=\left[\begin{array}{cccc}
-64528041013590 & 22316660983540 & -14660675253070 & -23287047712390 \\
-833898253680997 & -1831141920267418 & -628510813959081 & 1059071291894963 \\
509570466489060 & -4655727886202360 & -481050100124620 & -5658983802111740 \\
5843566312885470 & 3758022552321180 & -17342920355447690 & -1610120341214130
\end{array}\right]
$$

Next, from Algorithm 3 , we compute $Q=K J$,

$$
Q=\left[\begin{array}{cccc}
-64528041013590 & -236706501307159 & -105907161352079 & -149177855554037 \\
-833898253680997 & -3058966845644782 & -1368642151792744 & -1927830928699482 \\
509570466489060 & 1869243826365026 & 836336587427863 & 1178040248091503 \\
5843566312885470 & 21435799310693196 & 9590799761610551 & 13509331410827634
\end{array}\right]
$$

From the first row of matrix $Q$, it yields the values for $k, d_{1}, d_{2}$ and $d_{3}$ as follows:

$$
\begin{aligned}
k & =64528041013590, d_{1}=236706501307159, \\
d_{2} & =105907161352079, d_{3}=149177855554037 .
\end{aligned}
$$

Using Algorithm $3, \phi\left(N_{s}\right)=\frac{e_{s} d_{s}-1}{k}$ for $s=1,2,3$ can be computed as follows,

$$
\begin{aligned}
\phi\left(N_{1}\right)= & 23075243076701307224877654326747005767745347248898869640 \\
& 1562070407244760015236527680 \\
\phi\left(N_{2}\right)= & 4349917430502360609149739400310310943655109837893928735 \\
& 71099791027040771533822542568 \\
\phi\left(N_{3}\right)= & 9809146436233713823107593251565022080188392973725819800 \\
& 30651478359865012354849827664 .
\end{aligned}
$$

Next, from Algorithm $3 p_{s}^{r-1}$ for $s=1,2,3$ and $r=3$ can be computed as follows,

$$
\begin{aligned}
& p_{1}=954408180105791988011, p_{2}=770755872323270534549, \\
& p_{3}=994602670246108900363 .
\end{aligned}
$$

Finally, from Algorithm 3, $q_{s}$ for $s=1,2,3$ can be computed as follows,

$$
\begin{aligned}
& q_{1}=265426222155632917409, q_{2}=950015052524020374467, \\
& q_{3}=996970609016663314889 .
\end{aligned}
$$


This shows the factorization of 3 prime power moduli $N_{s}=p_{s}^{r} q_{s}$ for $s=$ $1,2,3$ and $r=3$ in polynomial time. Also, one can observe that our work yield $\min \left(d_{1}, d_{2}, d_{3}\right) \approx N^{0.1669}$ which is greater than $d \approx N^{0.1319}$, as reported in [14]. This shows that Shehu and Ariffin's attack can not yield the factorization of $t$ prime power moduli in this case.

3.2.3. The Attack on $t$ Prime Power Moduli $N_{s}=p_{s}^{r} q_{s}$ Satisfying System of Equation $e_{s} d-k_{s} \phi\left(N_{s}\right)=z_{s}$.

This section considers another case in which $t$ prime power moduli satisfies equations of the form $e_{s} d-k_{s} \phi\left(N_{s}\right)=z_{s}$ for unknown positive integers $d, k_{s}$, and $z_{s}$ for $s=1, \ldots, t$.

Taking $r \geq 2$, let $N_{s}=p_{s}^{r} q_{s}, s=1, \ldots, t$. The attack works for $t$ instances $\left(N_{s}, e_{s}\right)$ when there exists integer $d$ and $t$ integers $k_{s}$ such that $e_{s} d-k_{s} \phi\left(N_{s}\right)=z_{s}$ is satisfied. The attack shows that $t$ prime factors $p_{s}$ and $q_{s}$ of $t$ prime power moduli $N_{s}=p_{s}^{r} q_{s}$ for $s=1, \ldots, t$ can be found efficiently for $N=\max \left\{N_{s}\right\}$ and $d<N^{\varrho}, k_{s}<N^{\varrho}, z_{s}<N^{\varrho}$, for all $\varrho=\frac{t(1-\beta)}{t+1}$ for $0<\varrho \leq \frac{1}{2}$ and $o<\beta<1$. In this case, the instances $\left(N_{s}, e_{s}\right)$ shared common decryption exponent $d$.

Theorem 3.5. Let $N_{s}=p_{s}^{r} q_{s}$ be $t$ prime power moduli for $r \geq 2$ where $p_{s}$ and $q_{s}$ are prime numbers for $s=1 \ldots, t$. Let $\left(e_{s}, N_{s}\right)$ be public key pair and $\left(d, N_{s}\right)$ be private key pair with condition $e_{s}<\phi\left(N_{s}\right)$ and relation $e_{s} d \equiv z_{s} \bmod \phi\left(N_{s}\right)$ is satisfied. Let $N=\max \left\{N_{s}\right\}$. If there exists positive integer $d<N^{\varrho}$, $t$ integers $k_{s}<N^{\varrho}$ and $z_{s}<N^{\varrho}$, for all $\varrho=\frac{t(1-\beta)}{t+1}$ such that equation $e_{s} d-k_{s} \phi\left(N_{s}\right)=z_{s}$ holds, then prime factors $p_{s}$ and $q_{s}$ of $t$ prime power moduli $N_{s}$ can be successfully recovered in polynomial time for $0<\varrho \leq \frac{1}{2}$ and $o<\beta<1$.

Proof. Suppose $N_{s}=p_{s}^{r} q_{s}$ be $t$ prime power moduli, $N=\max \left\{N_{s}\right\}$ and $k_{s}<N^{\varrho}$ for $r \geq 2$ and $s=1, \ldots, t$. Then equation $e_{s} d-k_{s} \phi\left(N_{s}\right)=z_{s}$ can be rewritten as:

$$
e_{s} d-k_{s}\left(N_{s}-\left(N_{s}-\phi\left(N_{s}\right)\right)=z_{s}\right.
$$

Let $\triangle=2^{\frac{2 r+1}{r+1}} N_{s}^{\frac{r}{r+1}}$

$$
\begin{gathered}
e_{s} d-k_{s}\left(N_{s}-\triangle+\triangle-\left(N_{s}-\phi\left(N_{s}\right)\right)\right)=z_{s} \\
e_{s} d-k_{s}\left(N_{s}-\triangle\right)=z_{s}-k_{s}\left(N_{s}-\phi\left(N_{S}\right)-\triangle\right) \\
\left|\frac{e_{s}}{N_{s}-\triangle} d-k_{s}\right|=\frac{\left|z_{s}-k_{s}\left(N_{s}-\phi\left(N_{S}\right)-\triangle\right)\right|}{N_{s}-\triangle} .
\end{gathered}
$$

Since $N=\max \left\{N_{S}\right\}$ and $k_{s}<N^{\varrho}, z_{s}<N^{\varrho}$ are positive integers. Observe

$$
\begin{aligned}
\left|N_{s}-\phi\left(N_{S}\right)-\triangle\right| & <N_{s}^{\beta}<N^{\beta} \\
N_{s}-\triangle> & \frac{\sqrt{r+1}}{r} N
\end{aligned}
$$


for $\beta \in(0,1)$. Then plugging the conditions into equation $(3.2)$ yields

$$
\begin{aligned}
\frac{\left|z_{s}-k_{s}\left(N_{s}-\phi\left(N_{S}\right)-\triangle\right)\right|}{N_{s}-\triangle} & \leq \frac{\left|z_{s}+k_{s}\left(N_{s}-\phi\left(N_{S}\right)-\triangle\right)\right|}{N_{s}-\triangle} \\
& <\frac{N^{\varrho}+N^{\varrho}\left(N^{\beta}\right)}{\frac{\sqrt{r+1}}{r} N} \\
& =\frac{r\left(N^{\varrho}+N^{\varrho+\beta}\right)}{\sqrt{r+1} N} \\
& <\sqrt{2 r+1} N^{\varrho+\beta-1} .
\end{aligned}
$$

Hence,

$$
\left|\frac{e_{s}}{N_{s}-\triangle} d-k_{s}\right|<\sqrt{2 r+1} N^{\varrho+\beta-1} .
$$

We proceed to show the existence of an integer $d$, let $\varepsilon=\sqrt{2 r+1} N^{\varrho+\beta-1}$, for $\varrho=\frac{t(1-\beta)}{t+1}$. Then it gives

$$
N^{\varrho} \varepsilon^{t}=N^{\varrho}\left(\sqrt{2 r+1} N^{\varrho+\beta-1}\right)^{t}=(\sqrt{2 r+1})^{t} N^{\varrho+\varrho t+\beta t-t}=(2 r+1)^{\frac{t}{2}} .
$$

Following Theorem 2.4 $(2 r+1)^{\frac{t}{2}}<2^{\frac{t(t-3)}{4}} \cdot 3^{t}$ for $r, t \geq 2$, then it gives $N^{\varrho} \varepsilon^{t}<$ $2^{\frac{t(t-3)}{4}} \cdot 3^{t}$. It follows that if $d<N^{\varrho}$, then $d<2^{\frac{t(t-3)}{4}} \cdot 3^{t} \cdot \varepsilon^{-t}$ for $s=1, \ldots, t$, yields

$$
\left|\frac{e_{s}}{N_{s}-\triangle} d-k_{s}\right|<\varepsilon
$$

This clearly satisfies the conditions of Theorem 2.4 and proceeds to reveal the private key $d$ and $t$ integers $k_{s}$ for $s=1, \ldots, t$. Next, from $e_{s} d-k_{s} \phi\left(N_{s}\right)=z_{s}$, we make the following computations:

$$
\begin{aligned}
\phi\left(N_{s}\right) & =\frac{e_{s} d-z_{s}}{k_{s}} \\
p_{s}^{r-1} & =\operatorname{gcd}\left(\phi\left(N_{s}\right), N_{s}\right) \\
q_{s} & =\frac{N_{s}}{p_{s}^{r}} .
\end{aligned}
$$

Finally, the prime factors $p_{s}$ and $q_{s}$ can be revealed which lead to the factorization of $t$ prime power moduli $N_{s}=p_{s}^{r} q_{s}$ for $r \geq 2$ and $s=1, \ldots, t$ in polynomial time.

Let

$$
\begin{aligned}
X_{1} & =\frac{e_{1}}{N_{1}-2^{\frac{2 r+1}{r+1}} N_{1}^{\frac{r}{r+1}}}, X_{2}=\frac{e_{2}}{N_{2}-2^{\frac{2 r+1}{r+1}} N_{2}^{\frac{r}{r+1}}}, \\
X_{3} & =\frac{e_{3}}{N_{3}-2^{\frac{2 r+1}{r+1}} N_{3}^{\frac{r}{r+1}}} .
\end{aligned}
$$

Define,

$$
T=\left[3^{t+1} \times 2^{\frac{(t+1)(t-4)}{4}} \times \varepsilon^{-t-1}\right] .
$$

Consider the lattice $\mathcal{L}$ spanned by the matrix, 


$$
M=\left[\begin{array}{cccc}
1 & -\left[T\left(X_{1}\right)\right] & -\left[T\left(X_{2}\right)\right] & -\left[T \times X_{3}\right] \\
0 & T & 0 & 0 \\
0 & 0 & T & 0 \\
0 & 0 & 0 & T
\end{array}\right]
$$

Taking $r \geq 2$, the matrix $M$ can be used in computing the reduced basis after applying the LLL algorithm.

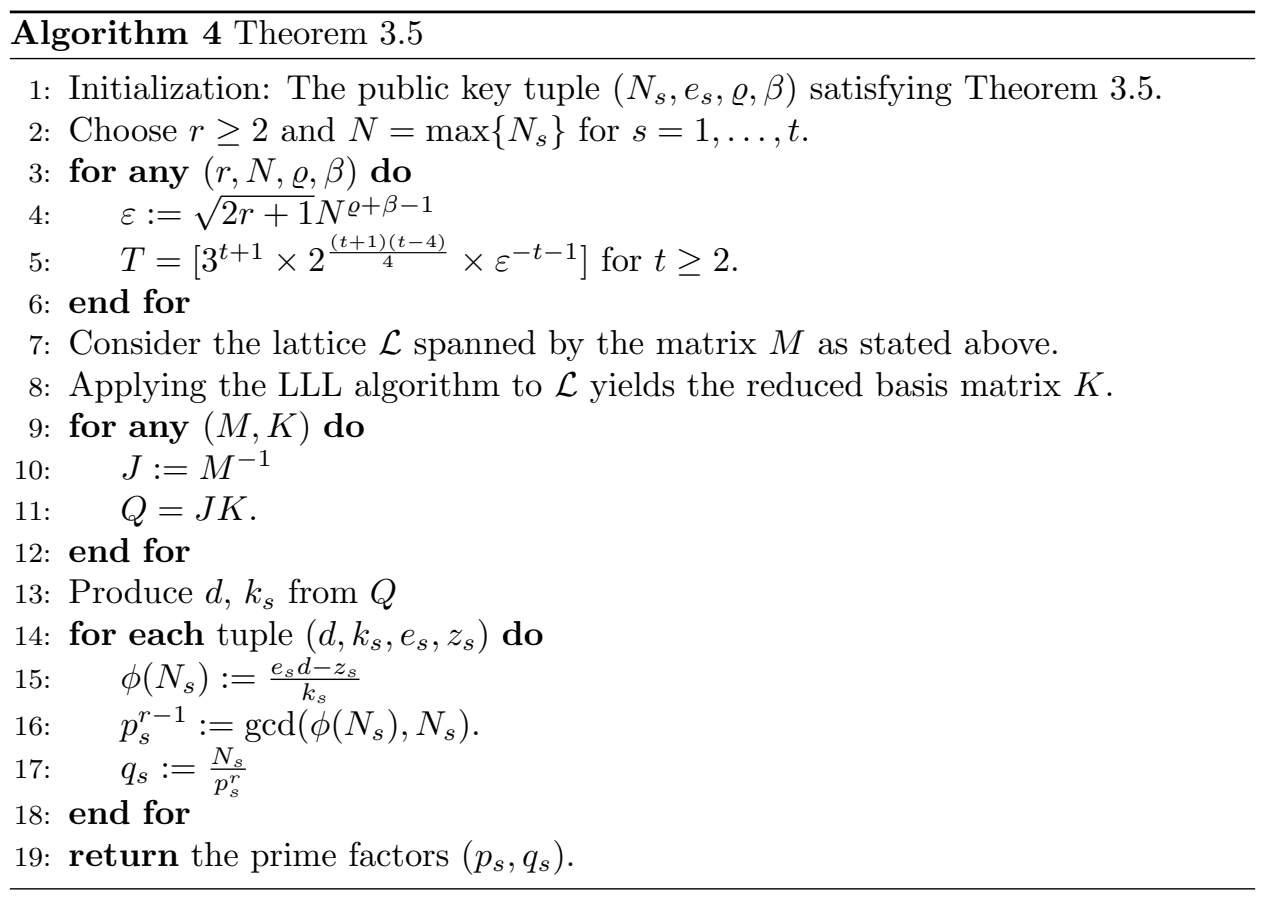

Example 3.4. This example gives an illustration of how Theorem 3.5 works on 3 prime power moduli and their corresponding public exponents:

$$
\begin{aligned}
& \text { Let } N_{1}=5525890830792963955829635376372589877105029843972435328080 \\
& 96725056837793945542263311852509300451 \\
& N_{2}=409009336956200004848526206159753677602922864786417839660 \\
& 443537581155477440303212646889912922681 \\
& N_{3}=1856599915884947721902864900852488867958645847203065789382 \\
& 29732631116403569017708089856336697379 \\
& e_{1}=535660672991610223946156685795497788662147614731651359062338 \\
& 800947852331357924924200110870181597 \\
& e_{2}=39814664618572247441461535731683170237273038362112873671207 \\
& 7969303212977619132096891312620129374 \\
& e_{3}=20945136845011188204703189941036359552207329716772570248911 \\
& 704906472236104028384132724576188013 .
\end{aligned}
$$


Observe $N=\max \left\{N_{1}, N_{2}, N_{3}\right\}$

$$
\begin{aligned}
N= & 55258908307929639558296353763725898771050298439724353280809 \\
& 6725056837793945542263311852509300451 .
\end{aligned}
$$

Using Algorithm 4 for $t=3 r=3$ and $\beta=0.75$ gives $\varrho=\frac{t(1-\beta)}{t+1}=0.1875$ and $\varepsilon=\sqrt{7} N^{\gamma+\beta-1}=0.000002745673398$.

Applying Theorem 2.4 and using Algorithm 4 for $n=t=3$, we compute

$$
T=\left[3^{t+1} \cdot 2^{\frac{(t+1)(t-4)}{4}} \cdot \varepsilon^{-t-1}\right]=712622481500000000000000 .
$$

Consider the lattice $\mathcal{L}$ spanned by the matrix,

$$
M=\left[\begin{array}{cccc}
1 & -\left[T\left(X_{1}\right)\right] & -\left[T\left(X_{2}\right)\right] & -\left[T \times X_{3}\right] \\
0 & T & 0 & 0 \\
0 & 0 & T & 0 \\
0 & 0 & 0 & T
\end{array}\right]
$$

Therefore, by applying the LLL algorithm to $\mathcal{L}$, it yields the reduced basis with the following matrix

$K=\left[\begin{array}{cccc}240575049922396781 & 45168467894653961 & -38707598398094518 & 195092642719432714 \\ -151248559543325924 & -488046878955018644 & 707575095426884472 & 620320224274381944 \\ -574956937086078187 & 491431650385399953 & -786566357699210214209783791280367322 \\ -89655203762229785 & -1063060408191942085 & -430492105618860770168843844246479710\end{array}\right]$

Next, from Algorithm 4, we compute $Q=K J$,

$Q=\left[\begin{array}{cccc}240575049922396781 & 233205101389831407 & 234185727874526165 & 27140351020204693 \\ -151248559543325924 & -146615102749620456 & -147231618648953995 & -17063028766409243 \\ -574956937086078187 & -557343294124629361 & -559686920366201207 & -64863472330366436 \\ -89655203762229785 & -86908641981956074 & -87274092461248535 & -10114405885730059\end{array}\right]$

From the first row of matrix $Q$, it yields the values for $k, d_{1}, d_{2}$ and $d_{3}$ as follows:

$$
\begin{aligned}
d & =240575049922396781, \quad k_{1}=233205101389831407, \\
k_{2} & =234185727874526165, \quad k_{3}=27140351020204693 .
\end{aligned}
$$

Using Algorithm $4, \phi\left(N_{s}\right)=\frac{e_{s} d-z_{s}}{k_{s}}$ for $s=1,2,3$ can be computed as follows, where $z_{1}, z_{2}, z_{3}$ are :

$$
\begin{aligned}
z_{1}= & 12594844191468409, z_{2}=7690976311642434, z_{3}=18446004731332273 \\
\phi\left(N_{1}\right)= & 5525890830792963955829616358238193471847011236132861263613349 \\
& 90579765661347407538020846597914464 \\
\phi\left(N_{2}\right)= & 4090093369562000048485248259766212750303796455582890471317695 \\
& 73374160014405441556077741716001204 \\
\phi\left(N_{3}\right)= & 1856599915884947721902855514033279681481441962537377493683119 \\
& 56715037950804743239281977597971160 .
\end{aligned}
$$


Next, from Algorithm 4, $p_{s}^{r-1}$ for $s=1,2,3$ and $r=3$ can be computed as follows,

$$
\begin{aligned}
& p_{1}=1121052815618170503691307, p_{2}=988706976202053289655339, \\
& p_{3}=901538558587875149528599 .
\end{aligned}
$$

Finally, from Algorithm 4, $q_{s}$ for $s=1,2,3$ can be computed as follows,

$$
\begin{aligned}
& q_{1}=392214892049653107897457, q_{2}=423185157151460671796099, \\
& q_{3}=253375960517480945644421 .
\end{aligned}
$$

This shows the factorization of 3 prime power moduli $N_{s}=p_{s}^{r} q_{s}$ simultaneously for $r \geq 2$ and $s=1, \ldots, t$. From our result, one can also observe that our work yields $d \approx N^{0.18154}$. The equation $e_{s} d-k_{s} \phi\left(N_{s}\right)=z_{s}$ is a generalization of equation $e_{i} d-k_{i} \phi\left(N_{i}\right)=1$, as reported in [14].

3.2.4. The Attack on $t$ Prime Power Moduli $N_{s}=p_{s}^{r} q_{s}$ Satisfying System of Equation $e_{s} d_{s}-k \phi\left(N_{s}\right)=z_{s}$.

This section presents another cryptanalysis attack in which $t$ prime power moduli $N_{s}=p_{s}^{r} q_{s}$ satisfies equations of the form $e_{s} d_{s}-k \phi\left(N_{s}\right)=z_{s}$ for unknown positive integers $d_{s}, k$, and $z_{s}$ for $s=1, \ldots, t$ and $r \geq 2$ which can be simultaneously factored in polynomial time. In this case, every pair of the instances $\left(N_{s}, e_{s}\right)$ has its own unique decryption exponent $d_{s}$.

Theorem 3.6. Let $N_{s}=p_{s}^{r} q_{s}$ be $t$ prime power moduli where $p_{s}$ and $q_{s}$ are prime numbers for $s=1, \ldots, t$ and $t \geq 3$. Let $\left(e_{s}, N_{s}\right)$ be public key pair and $\left(d_{s}, N_{s}\right)$ be private key pair with $e_{s}<\phi\left(N_{s}\right)$ and relation $e_{s} d_{s} \equiv z_{s} \bmod \phi\left(N_{s}\right)$ is satisfied. Let $e=\min \left\{e_{s}\right\}=N^{\alpha}$ be public exponent. If there exists positive $t$ integers $d_{s}<$ $N \varrho$, integer $k<N^{\varrho}$ and $t$ integers $z_{s}<N \varrho$, for all $\varrho=\frac{t(\alpha-\beta)}{t+1}$ such that equation $e_{s} d_{s}-k \phi\left(N_{s}\right)=z_{s}$ holds, then prime factors $p_{s}$ and $q_{s}$ of $t$ prime power moduli $N_{s}=p_{s}^{r} q_{s}$ for $N_{s}$ and $r \geq 2$ can be successfully recovered in polynomial time for $0<\varrho \leq \frac{1}{2}, 0<\beta<1$ and $\beta<\alpha<1$.

Proof. Suppose $N_{s}=p_{s}^{r} q_{s}$ be $t$ prime power moduli and $e=\min \left\{e_{s}\right\}=N^{\alpha}$ be public exponent for $s=1, \ldots t$ and suppose that $d_{s}<N^{\varrho}$, for $r \geq 2$ and $t \geq 3$. Then equation $e_{s} d_{s}-k \phi\left(N_{s}\right)=z_{s}$ can be rewritten as

$$
e_{s} d_{s}-k\left(N_{s}-\left(N_{s}-\phi\left(N_{s}\right)\right)\right)=z_{s} .
$$

Let $\triangle=2^{\frac{2 r+1}{r+1}} N_{s}^{\frac{r}{r+1}}$

$$
\begin{aligned}
e_{s} d_{s}-k\left(N_{s}-\triangle+\triangle-\left(N_{s}-\phi\left(N_{s}\right)\right)\right) & =z_{s} \\
e_{s} d_{s}-k\left(N_{s}-\triangle\right) & =z_{s}-k\left(N_{s}-\phi\left(N_{s}\right)-\triangle\right) \\
\left|k \frac{\left(N_{s}-\triangle\right)}{e_{s}}-d_{s}\right| & =\frac{\left|z_{s}-k\left(N_{s}-\phi\left(N_{s}\right)-\triangle\right)\right|}{e_{s}} .
\end{aligned}
$$

Since $N=\max \left\{N_{s}\right\}$ and $d_{s}<N^{\varrho}, k<N^{\varrho}, z_{s}<N^{\varrho}$. Observe

$$
\left|N_{s}-\phi\left(N_{s}\right)-\triangle\right|<N_{s}^{\beta}<N^{\beta}
$$

for $\beta \in(0,1)$. Also since $e=\min \left\{e_{s}\right\}=N^{\alpha}$, for $s=1, \ldots t$ then it gives 


$$
\begin{aligned}
\frac{\left|z_{s}-k\left(N_{s}-\phi\left(N_{s}\right)-\triangle\right)\right|}{e_{s}} \leq & \frac{\left|z_{s}+k\left(N_{s}-\phi\left(N_{s}\right)-\triangle\right)\right|}{e_{s}} \\
& <\frac{N^{\varrho}+N^{\varrho}\left(N^{\beta}\right)}{N^{\alpha}} \\
& =\frac{N^{\varrho}+N^{\varrho+\beta}}{N^{\alpha}} \\
& <\sqrt{r+2} N^{\varrho+\beta-\alpha} .
\end{aligned}
$$

Hence,

$$
\left|k \frac{\left(N_{s}-\triangle\right)}{e_{s}}-d_{s}\right|<\sqrt{r+2} N^{\varrho+\beta-\alpha} .
$$

We proceed to show the existence of integer $k$ and $t$ integers $d_{s}$. Let $\varepsilon=\sqrt{r+2} N^{\varrho+\beta-\alpha}$ and $\varrho=\frac{t(\alpha-\beta)}{t+1}$. Then it gives

$$
N^{\varrho} \varepsilon^{t}=N^{\varrho}\left(\sqrt{r+2} N^{\varrho+\beta-\alpha}\right)^{t}=(\sqrt{r+2})^{t} N^{\varrho+\beta t-\alpha t}=(r+2)^{\frac{t}{2}} .
$$

Following Theorem 2.4. $(r+2)^{\frac{t}{2}}<2^{\frac{t(t-3)}{4}} \cdot 3^{t}$ for $r, t \geq 2$, then $N^{\varrho} \varepsilon^{t}<2^{\frac{t(t-3)}{4}} \cdot 3^{t}$. It follows that if $k<N^{\varrho}$ then $k<2^{\frac{t(t-3)}{4}} \cdot 3^{t} \cdot \varepsilon^{-t}$ for $s=1, \ldots, t$, yields

$$
\left|k \frac{\left(N_{s}-\triangle\right)}{e_{s}}-d_{s}\right|<\varepsilon \text {. }
$$

This clearly satisfies the conditions of Theorem 2.4 and proceeds to reveal the private keys $t$ integers $d_{s}$ and $k$ for $s=1, \ldots, t$. Next, from $e_{s} d_{s}-k \phi\left(N_{s}\right)=z_{s}$ we make the following computations:

$$
\begin{aligned}
\phi\left(N_{s}\right) & =\frac{e_{s} d_{s}-z_{s}}{k} \\
p_{s}^{r-1} & =\operatorname{gcd}\left(\phi\left(N_{s}\right), N_{s}\right) \\
q_{s} & =\frac{N_{s}}{p_{s}^{r}} .
\end{aligned}
$$

Finally, the prime factors $p_{s}$ and $q_{s}$ can be revealed which lead to the factorization of $t$ prime power moduli $N_{s}$ for $s=1, \cdots, t$ in polynomial time.

Let

$X_{1}=\frac{\left(N_{1}-2^{\frac{2 r+1}{r+1}} N_{1}^{\frac{r}{r+1}}\right)+1}{e_{1}}, X_{2}=\frac{\left(N_{2}-2^{\frac{2 r+1}{r+1}} N_{2}^{\frac{r}{r+1}}\right)+1}{e_{2}}, X_{3}=\frac{\left(N_{3}-2^{\frac{2 r+1}{r+1}} N_{3}^{\frac{r}{r+1}}\right)+1}{e_{3}}$.

Define,

$$
T=\left[3^{t+1} \times 2^{\frac{(t+1)(t-4)}{4}} \times \varepsilon^{-t-1}\right] .
$$

Consider the lattice $\mathcal{L}$ spanned by the matrix,

$$
M=\left[\begin{array}{cccc}
\mathbf{1} & -\left[T\left(X_{1}\right)\right] & -\left[T\left(X_{2}\right)\right] & -\left[T\left(X_{3}\right)\right] \\
0 & T & 0 & 0 \\
0 & 0 & T & 0 \\
0 & 0 & 0 & T
\end{array}\right]
$$

Taking $r \geq 2$, the matrix $M$ can be used in computing the reduced basis after applying the LLL algorithm 


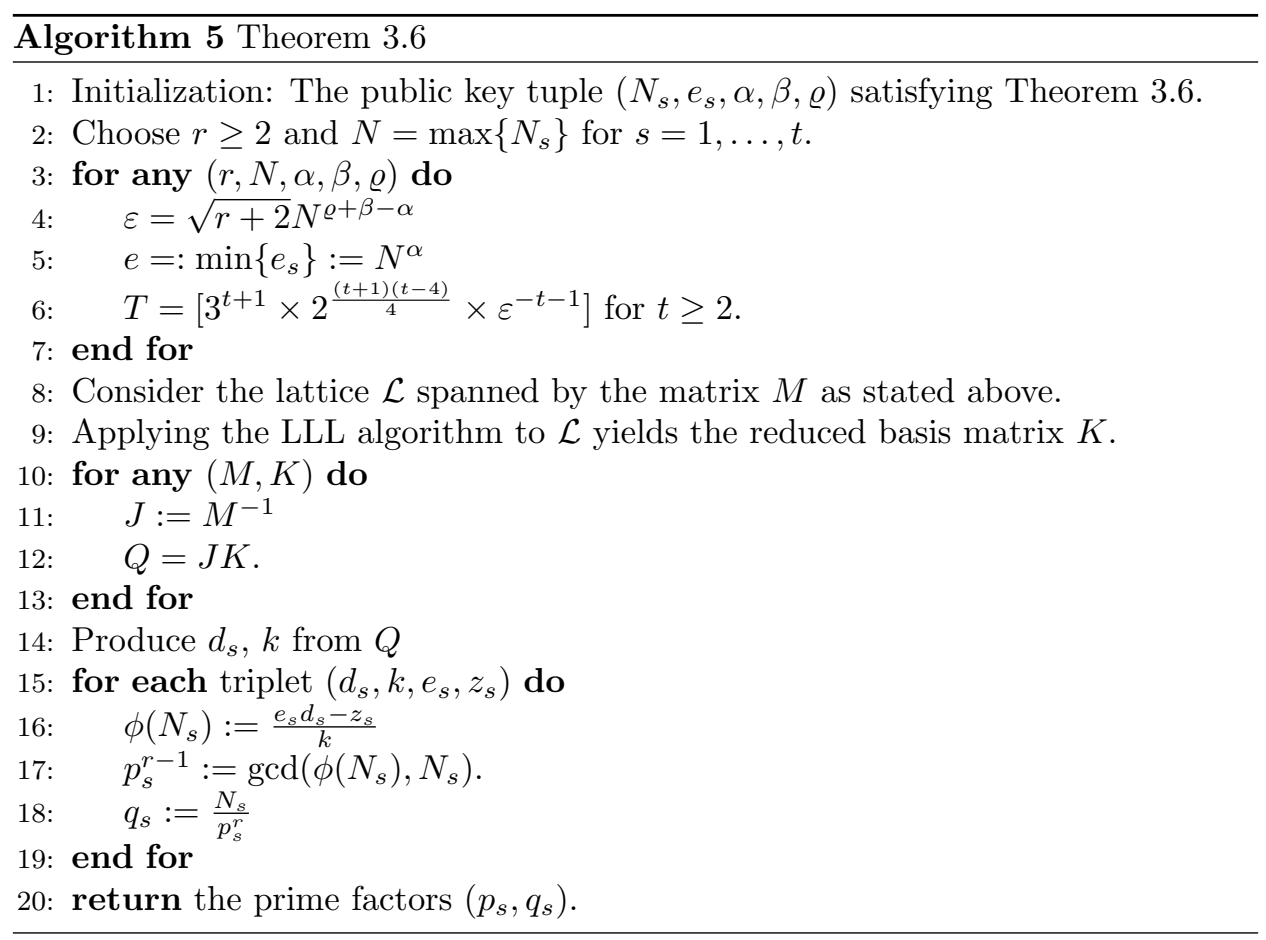

Example 3.5. This example gives an illustration of how Theorem 3.6 works on 3 prime power moduli and their corresponding public exponents:

$$
\begin{aligned}
N_{1}= & 118206700499027973555226065271614027133355822416165333781707131772561 \\
& 895107920252379 \\
N_{2}= & 1531872675863933704937871257817812503603379715206904363401447389746 \\
& 44921936384902153 \\
N_{3}= & 924899290347826697102573577323286044355305745566432529788292084959 \\
& 590562885708269169 \\
e_{1}= & 94472170189652409334810337024409313700966097954777781302492419150 \\
& 324241356533223123 \\
e_{2}= & 925995466598943224506439532320239713941284965387703819238402532 \\
& 25802616168922466797 \\
e_{3}= & 62982869619724355834375582908978776341707025327451907660592240 \\
& 8153811221100854704887 .
\end{aligned}
$$

Observe

$$
\begin{aligned}
N=\max \left\{N_{1}, N_{2}, N_{3}\right\}= & 92489929034782669710257357732328604435530574556643 \\
& 2529788292084959590562885708269169 \\
e=\min \left\{e_{1}, e_{2}, e_{3}\right\}= & 9259954665989432245064395323202397139412849653877038 \\
& 1923840253225802616168922466797
\end{aligned}
$$


with $e=\min \left\{e_{1}, e_{2}, e_{3}\right\}=N^{\alpha}$ for $\alpha=0.9880965575$. Taking $t=3, \beta=0.75$ it gives $\varrho=\frac{t(\alpha-\beta)}{t+1}=0.1785724181, \varepsilon=0.00002246340004$.

Applying Theorem 2.4 and using Algorithm 5, we compute

$$
C=\left[3^{t+1} \cdot 2^{\frac{(t+1)(t-4)}{4}} \cdot \varepsilon^{-t-1}\right]=159057099200000000000 .
$$

Consider the lattice $\mathcal{L}$ spanned by the matrix,

$$
M=\left[\begin{array}{cccc}
\mathbf{1} & -\left[T\left(X_{1}\right)\right] & -\left[T\left(X_{2}\right)\right] & -\left[T\left(X_{3}\right)\right] \\
0 & T & 0 & 0 \\
0 & 0 & T & 0 \\
0 & 0 & 0 & T
\end{array}\right]
$$

Therefore, by applying the LLL algorithm to $\mathcal{L}$, it yields the reduced basis with the following matrix

$$
K=\left[\begin{array}{cccc}
-192479622515690 & -477895645520 & -115255751942450 & 123625906116600 \\
300578256728925 & 1229934560231400 & -602650460625375 & 38597963550500 \\
-1282731106006613 & 354397216043096 & -475121843615865 & -2396325179490180 \\
1337229767906843 & -1481780789001256 & -3446686105094985 & -1015254431182020
\end{array}\right]
$$

Next, from Algorithm 5, we compute $Q=K J$,

$$
Q=\left[\begin{array}{cccc}
-192479622515690 & -240836862805235 & -318418701848887 & -282655057392665 \\
300578256728925 & 376093445279694 & 497246082783771 & 441397189459762 \\
-1282731106006613 & -1604995538518056 & -2122019818292808 & -1883682177099557 \\
1337229767906843 & 1673186064806441 & 2212176859064010 & 1963713103001738
\end{array}\right]
$$

From the first row of matrix $Q$, it yields the values for $k, d_{1}, d_{2}$ and $d_{3}$ as follows:

$$
\begin{aligned}
k & =192479622515690, d_{1}=240836862805235, \\
d_{2} & =318418701848887, d_{3}=282655057392665 .
\end{aligned}
$$

Using Algorithm $5, \phi\left(N_{s}\right)=\frac{e_{s} d_{s}-z_{s}}{k}$ for $s=1,2,3$ can be computed as follows, where $z_{1}, z_{2}, z_{3}$ are :

$$
\begin{aligned}
& z_{1}=125587188015385, z_{2}=213104320451339, z_{3}=223377252772855 \\
& \phi\left(N_{1}\right)= 1182067004990279735548122132240889792044275182121851 \\
& 20438299120180310861269069393208 \\
& \phi\left(N_{2}\right)= 153187267586393370493317462323916741449295154485594 \\
& 324234978653995475779166247995440 \\
& \phi\left(N_{3}\right)= 92489929034782669710021054527475913748082630652189 \\
& 3518875458562989447638863244024900 .
\end{aligned}
$$

Next, from Algorithm $5 p_{s}^{r-1}$ for $s=1,2,3$ and $r=3$ can be computed as follows,

$$
\begin{aligned}
& p_{1}=601114833736581054997, p_{2}=601114833736581054997, \\
& p_{3}=1161433282369002470551 .
\end{aligned}
$$


Finally, from Algorithm 5 $q_{s}$ for $s=1,2,3$ can be computed as follows,

$$
\begin{aligned}
& q_{1}=544214062088679626023, q_{2}=718297824560170977461, \\
& q_{3}=590352823628934085319 .
\end{aligned}
$$

This shows the factorization of 3 prime power moduli $N_{s}=p_{s}^{r} q_{s}$ simultaneously for $r \geq 2$ and $s=1, \ldots, t$. From our result, one can also observe that our work yields $\min \left(d_{1}, d_{2}, d_{3}\right) \approx N^{0.1712}$. The equation $e_{s} d_{s}-k \phi\left(N_{s}\right)=z_{s}$ is a generalization of equation $e_{i} d_{i}-k \phi\left(N_{i}\right)=1$, as reported in [14.

\section{Conclusion}

In this paper, we developed new technique that led to the successful factorization of prime power modulus $N=p^{r} q$ for $r \geq 2$ via good approximation of $\phi(N)$. The paper also showed that using $N-\left[2^{\frac{2 r+1}{r+1}} N^{\frac{r}{r+1}}\right]$ as good approximation of $\phi(N)$ led to the extension of the bound to susceptible decryption exponent. The paper also presented four cryptanalysis attacks that successfully factored $t$ prime power moduli $N_{s}=p_{s}^{r} q_{s}$ for $s=1, \ldots, t$ using generalized key equations of the form $e_{s} d-k_{s} \phi\left(N_{s}\right)=1, e_{s} d_{s}-k \phi\left(N_{s}\right)=1, e_{s} d-k_{s} \phi\left(N_{s}\right)=z_{s}$ and $e_{s} d_{s}-k \phi\left(N_{s}\right)=z_{s}$. It has improved susceptible decryption exponent bounds of 14 from $d \approx N^{0.1857}$ to $d \approx N^{0.1863}$ and from $\min \left\{d_{i}\right\} \approx N^{0.1319}$ to $\min \left\{d_{s}\right\} \approx N^{0.1669}$. From these results, the paper generalized key equations of [14] from $e_{i} d-k_{\phi}\left(N_{i}\right)=1$ to $e_{s} d-k_{s} \phi\left(N_{s}\right)=$ $z_{s}$ and also from $e_{i} d_{i}-k \phi\left(N_{i}\right)=1$ to $e_{s} d_{s}-k \phi\left(N_{s}\right)=z_{s}$.

\section{REFERENCES}

[1] A. Rivest, R. Shamir, L. Adleman, A method for obtaining digital signatures and public-key cryptosystems, Communications of the ACM 212 (1978) 120-126. .

[2] M. K. Dubey, N. Ratan, R. Verma, N. Saxena, Cryptanalytic attacks and countermeasures on RSA, in: P. K. (2014), In Proceedings of the Third International Conference on Soft Computing for Problem Solving, Springer, (2014), 10-18. .

[3] T. Fujioka, A. Okamoto, S. Miyaguchi, ESIGN, An effient digital signature implementation for smart cards, Advances in Cryptology EUROCRYPT 91, Lecture Notes in Computer Science, Springer, (1991), 446-457.

[4] T. Okamoto, S. Uchiyama, A new public-key cryptosystem as secure as factoring, in: Advances in Cryptology EUROCRYPT'98, Lecture Notes in Computer Science, Springer, (1998), 308-318.

[5] T. Takagi, Fast RSA-type cryptosystem modulo $p^{k} q$, in: Advances in Cryptology CRYPTO '98. CRYPTO 1998, Lecture Notes in Computer Science, Springer, (1998), 318-326.

[6] A. May, Secret exponent attacks on RSA-type schemes with moduli $N=p^{r} q$, in: Public Key Cryptography-PKC 2004, Springer, (2004), 218-230.

[7] S. Sarkar, Small secret exponent attack on RSA variant with modulus $N=p^{2} q$, in: Proceedings International Workshop on Coding and Cryptography-WCC2013 Norway and INRIA, (2013), 215-222.

[8] R. Lu, Y. Zhang, D. Lin, New results on solving linear equations modulo unknown divisors and its applications, IACR Cryptology eprint 1 (2014) 343-354.

[9] S. Sarkar, Revisiting prime power RSA, Discrete Applied Mathematics 203(C) (2016) 127133.

[10] K. Itoh, K. Kunihiro, K. Kurosawa, Small secret key attack on a variant of RSA (due to takagi), in: CT-RSA 2008, in:LNCS, (2008), 387-406.

[11] J. Blomer, A. May, A generalized Wiener attack on RSA, in: International Workshop on Public Key Cryptography, Springer, (2004), 1-13.

[12] J. Hinek, On the security of some variants of RSA, Phd thesis, Universiti Waterloo, Ontario, Canada (2007). 
[13] A. Nitaj, M. Ariffin, D. Nassr, H. Bahig, New Attacks on the RSA cryptosystem, in: Progress in Cryptology AFRICACRYPT 2014. Lecture Notes in Computer Science, 8469, Springer, (2014), 178-198.

[14] S. Shehu, M.R.K Ariffin, New attacks on prime power $R S A N=p^{r} q$ using good approximation of $\phi(N)$, Malaysian Journal of Mathematical Sciences special issues:The 5th International Cryptology and Information Security Conference(New Ideas in ) 11(S) (2017) $121-138$.

[15] H. Lenstra, A.K. Lenstra, L. Lovsz, Factoring polynomials with rational coefficients, Mathematische Annalen (1982) 513-534.

[16] A. Nitaj, Diophantine and lattice cryptanalysis of the RSA cryptosystem, in: Artificial Intelligence, Evolutionary Computing and Metaheuristics, Springer, (2013), 139-168.

[17] X. Wang, X. G., M. Wang, X. Meng, Mathematical Foundations of Public Key Cryptography, CRC Press, Boca Rating London New York, 2016.

Saidu Isah Abubakar,

Department of Mathematics, Sokoto State University, Sokoto, :+2348069191131, Orcid NUMBER:0000-0002-0201-0064

Email address: siabubakar82@gmail.com

ZAID IBRAHI,

Department of Mathematics, Sokoto State University, Sokoto,+2348035780166 : Orcid NUMBER:0000-0002-0251-6495

Email address: malamzaid2@gmail.com

SADiq Shehu,

Department of Mathematics, Sokoto State University, Sokoto, +2348066284440: Orcid NUMBER:0000-0001-5908-7452

Email address: sadiqshehuzezi@gmail.com

Ahmad Rufai,

Department of Mathematics, Sokoto State University, Sokoto, +2347068272590: Orcid NUMBER:0000-0003-3223-9924

Email address: rufaiahmad35@yahoo.com 